\title{
Particle Acceleration and Heating in Regions of Magnetic Flux Emergence
}

\author{
H. Isliker ${ }^{1}\left(\mathbb{1}\right.$, V. Archontis $^{2}$ (i), and L. Vlahos ${ }^{1}$ (i) \\ ${ }^{1}$ Section of Astrophysics, Astronomy and Mechanics, Physics Department, Aristotle University, Thessaloniki, GR 541 24, Greece; isliker@astro.auth.gr, \\ vlahos@ astro.auth.gr
${ }^{2}$ School of Mathematics and Statistics, University of St Andrews, North Haugh, St Andrews, Fife KY16 9SS, UK; vasilis@mcs.st-and.ac.uk \\ Received 2019 May 25; revised 2019 July 7; accepted 2019 July 8; published 2019 September 2
}

\begin{abstract}
The interaction between emerging and pre-existing magnetic fields in the solar atmosphere can trigger several dynamic phenomena, such as eruptions and jets. A key element during this interaction is the formation of largescale current sheets, and eventually their fragmentation that leads to the creation of a strongly turbulent environment. In this paper, we study the kinetic aspects of the interaction (reconnection) between emerging and ambient magnetic fields. We show that the statistical properties of the spontaneously fragmented and fractal electric fields are responsible for the efficient heating and acceleration of charged particles, which form a power-law tail at high energies on sub-second timescales. A fraction of the energized particles escapes from the acceleration volume, with a super-hot component with a temperature close to $150 \mathrm{MK}$, and with a power-law high-energy tail with an index between -2 and -3 . We estimate the transport coefficients in energy space from the dynamics of the charged particles inside the fragmented and fractal electric fields, and the solution of a fractional transport equation, as appropriate for a strongly turbulent plasma, agrees with the test-particle simulations. We also show that the acceleration mechanism is not related to Fermi acceleration, and the Fokker-Planck equation is inconsistent and not adequate as a transport model. Finally, we address the problem of correlations between spatial transport and transport in energy space. Our results confirm the observations reported for high-energy particles (hard X-rays, type III bursts, and solar energetic particles) during the emission of solar jets.
\end{abstract}

Unified Astronomy Thesaurus concepts: Solar energetic particles (1491); Solar magnetic flux emergence (2000); Solar magnetic reconnection (1504); Magnetohydrodynamics (1964)

\section{Introduction}

Emerging magnetic flux is one of the mechanisms responsible for the formation of large-scale reconnecting current sheets in the solar corona. The evolution of the reconnecting current sheet is proposed as the key mechanism for many explosive phenomena in the solar atmosphere, i.e., flares, prominence eruptions, jets, and coronal mass ejections (Heyvaerts et al. 1977; Archontis et al. 2004, 2005; Galsgaard et al. 2005; Archontis 2012; Archontis \& Hood 2012, 2013; Karimabadi \& Lazarian 2013; Moreno-Insertis \& Galsgaard 2013; Jiang et al. 2016; Raouafi et al. 2016; Wyper et al. 2016, 2017). Most of the cited studies focus on the large-scale formation of the current sheet, the complex magnetic environment around the unstable current structure, the ejected plasma, and the jets in the vicinity of the current sheet.

Several RHESSI observations of the base of coronal jets are associated with hard X-ray (HXR) emission (Bain \& Fletcher 2009; Glesener et al. 2012; Glesener \& Fleishman 2018). Frequently during coronal jets, the temporal profile of the associated HXRs matches the associated type III radio bursts (Chen et al. 2013). Impulsive solar energetic particle events are also related to the jets (see the review by Raouafi et al. 2016). It is then obvious that jets act as an efficient mechanism for the heating and acceleration of particles, mainly due to the reconnecting current sheets at the boundary between the emerging magnetic flux and the ambient magnetic field in the solar atmosphere.

In a series of separate studies the evolution of the unstable current sheets has been analyzed in detail using magnetohydrodynamic (MHD) and particle-in-cell (PIC) simulations. The main common observation is that the reconnecting current sheet will fragment, forming a very efficient particle acceleration environment (Onofri et al. 2006; Drake et al. 2006; Kowal et al. 2011; Hoshino 2012; Cargill et al. 2012; Lazarian et al. 2012; Baumann et al. 2013; Karimabadi \& Lazarian 2013; Guo et al. 2015). The results of these studies were limited to the analysis of the evolution of particles inside very small-scale PIC simulations with periodic boundary conditions, or test-particle simulations in the fields generated through the solution of the resistive MHD equations, or by using the Fokker-Planck equation with analytically estimated transport coefficients (Drake et al. 2013; Guo et al. 2015) or the comprehensive Fokker-Planck transport equations that were developed recently for studying energetic particle transport and acceleration in plasma regions containing numerous dynamic small-scale flux ropes (e.g., Zank et al. 2014; le Roux et al. 2015, 2018). Several recent studies based their analysis of the acceleration of particles in fragmented current sheets on the findings of PIC simulations or on simplified analytical models for the interaction of the particles with magnetic blobs resulting from the evolution of the current sheets. They assume that the fragments of the evolving current sheet are uniformly distributed in space, and the main acceleration mechanism is first-order Fermi acceleration in a periodic simulation box (Kowal et al. 2011; Lazarian et al. 2015), and in particular they consider acceleration by island contraction, either in a compressible (Zank et al. 2014; le Roux et al. 2015; Li et al. 2018, which indeed leads to a first-order Fermi process) or an area-preserving way (Dahlin et al. 2016, which actually results in a second-order Fermi process). All these assumptions are open for discussion when one considers the more realistic fragmentation of the large-scale current sheets in the solar corona, which are three-dimensional open systems, and when one relies on the dynamic evolution of the particle orbits in 
order to test the validity of the FP equation, as we do it in our approach.

In the present paper, we focus on the kinetic aspects of the interaction between an emerging and a pre-existing magnetic field in the solar corona. For this study, we use an MHD numerical simulation, similar to the work by Archontis \& Hood (2012, 2013), which shows: (i) the formation and fragmentation of large-scale current sheets during the emergence and interaction phase, and (ii) the emission of "standard" and more explosive eruption-driven "blowout" jets (Moore et al. 2010). Our analysis explores the statistical properties of the electric field in the fragmented reconnecting current sheets, and the resulting energy distribution of electrons, by using a testparticle numerical code. In our analysis, the particle dynamics inside the fragmented electric fields is directly used to estimate the transport coefficients of the particles, and we solve the transport equation that is appropriate for such an environment.

We address three important questions in this article:

1. What are the statistical properties of the electric fields associated with the fragmentation of the large-scale reconnecting current sheet?

2. What are the characteristics of the electron energy distribution driven by the fragmented electric fields ?

3. Are the transport properties of the electrons inside the fragmented current sheet "normal" or "anomalous"? (We consider both, energy- and position space.)

In Section 2, we briefly present the MHD model used in this study and the test-particle code. In Section 3, we analyze the statistical properties of the fragmented electric fields. In Section 4, we use the test-particle code to follow the evolution of the energy distribution in the vicinity of the standard jet, and in Section 5 we investigate with the same tools the region near the base of the blowout jet. In Sections 6 and 7 we examine the transport properties of the particles in energy space and in position space, respectively, and in Section 8 we summarize our results.

\section{The Model}

\subsection{The MHD Model}

For this model, we are using the Lare3d code (Arber et al. 2001). We solve the 3D time-dependent, resistive, and compressible MHD equations in Cartesian geometry, as in the model by Archontis et al. (2013). Initially, the plasma is embedded into a plane-parallel hydrostatic atmosphere. A highly stratified atmosphere in hydrostatic equilibrium is included in the model (see Figure 1). The atmosphere consists of various layers. The solar interior is modeled by an adiabatically stratified layer, which resides in the range $(-3.6 \mathrm{Mm} \leqslant z<0 \mathrm{Mm})$. Above it, the layer is isothermal $(5100 \mathrm{~K})$, then the temperature increases smoothly with height up to $\approx 3 \times 10^{4} \mathrm{~K}$. This layer represents the photosphere/ chromosphere and it is located at $0 \mathrm{Mm} \leqslant z<1.9 \mathrm{Mm}$. Then, at $1.9 \mathrm{Mm} \leqslant z \leqslant 3 \mathrm{Mm}$, the temperature increases with height, forming a layer that represents the transition region. The top layer in the stratified atmosphere, is an isothermal layer $(\mathcal{O}(1) \mathrm{MK})$ at $3 \mathrm{Mm}<z \leqslant 50.4 \mathrm{Mm}$, which is mimicking the solar corona. In the solar interior, at $z_{0}=-2.1 \mathrm{Mm}$, we have included a twisted magnetic flux tube oriented along the $Y$-axis. The tube's magnetic field is defined by

$$
B_{y}=B_{0} \exp \left(-r^{2} / R^{2}\right), B_{\theta}=\alpha r B_{y},
$$

where $B_{y}$ is the longitudinal component of the magnetic field (i.e., along the axis of the tube) and $B_{\theta}$ is the azimuthal component. $B_{0}=3.9 \mathrm{kG}$ is the initial field strength of the tube and $\alpha=2.2 \times 10^{-3} \mathrm{~km}^{-1}$ is the parameter associated with the uniform twist around the axis of the tube. With this twist, the tube is marginally stable to the kink instability. The radius of the tube is $R=450 \mathrm{~km}$ and $r$ is the radial distance from the axis of the tube $\left(r^{2}=x^{2}+\left(z+z_{0}\right)^{2}\right)$. To initiate the emergence of the tube, we impose a density along the axis of the tube so that its central part is more buoyant than its footpoints:

$$
\Delta \rho=\left[p_{t}(r) / p(z)\right] \rho(z) \exp \left(-y^{2} / \lambda^{2}\right),
$$

where $p_{t}$ is the pressure within the tube and $\lambda$ defines the length of the buoyant part of the emerging field. In this model, we use $\lambda=0.9 \mathrm{Mm}$.

The atmosphere in this model is magnetized. More precisely, there is an ambient magnetic field in the corona, with an oblique orientation, and it is defined by

$$
B_{c}=B_{c}(z)(0, \cos \theta, \sin \theta),
$$

where $\theta=80^{\circ}$ is the angle that the ambient field makes with respect to the positive $y$-axis (i.e., a vertical field has $\theta=90^{\circ}$ ). The relative orientation between the emerging field and the ambient field is such that effective reconnection occurs when the two fields come into contact. The strength of the ambient field is $B_{c}(z)=9 \mathrm{G}$ above the photosphere, and it gradually decreases to 0 under the solar surface.

The numerical domain is $[-23.4,23.4] \times[-25.2$, $25.2] \times[-3.6,50.4] \mathrm{Mm}$ in the transverse $(x)$, longitudinal $(y)$, and vertical $(z)$ directions, respectively. The numerical grid has 384 nodes in the transverse $(x)$ direction, 416 nodes in the $y$ direction, and 512 nodes along the height. Periodic boundary conditions have been implemented along the transverse and longitudinal directions, and open boundary conditions have been employed at the top of the numerical domain. The bottom boundary is a non-penetrating, perfectly conducting wall.

\subsection{The Kinetic Model}

The kinetic aspects of the dynamics of particles in an environment of fragmented current sheets are explored here by performing test-particle simulations in the electromagnetic fields of the MHD simulations. The relativistic guiding-center equations of motions are used in the test-particle simulations, as given in Tao et al. (2007), which rederive the relations of Grebogi \& Littlejohn (1984); and we reorder the expressions to bring the equations into the form of Hamamatsu et al. (2007),

$$
\begin{gathered}
\frac{d \boldsymbol{r}}{d t}=\frac{1}{B_{\|}^{*}}\left[\frac{u_{\|}}{\gamma} \boldsymbol{B}^{*}+\hat{\boldsymbol{b}} \times\left(\frac{\mu}{q \gamma} \nabla B-\boldsymbol{E}^{*}\right)\right], \\
\frac{d u_{\|}}{d t}=-\frac{q}{m_{0} B_{\|}^{*}} \boldsymbol{B}^{*} \cdot\left(\frac{\mu}{q \gamma} \nabla B-\boldsymbol{E}^{*}\right),
\end{gathered}
$$

with $\boldsymbol{r}$ being the particle position, and where $\boldsymbol{B}^{*}$ and $\boldsymbol{E}^{*}$ are the modified fields, defined by

$$
\boldsymbol{B}^{*}=\boldsymbol{B}+\frac{m_{0}}{q} u_{\|} \nabla \times \hat{\boldsymbol{b}},
$$




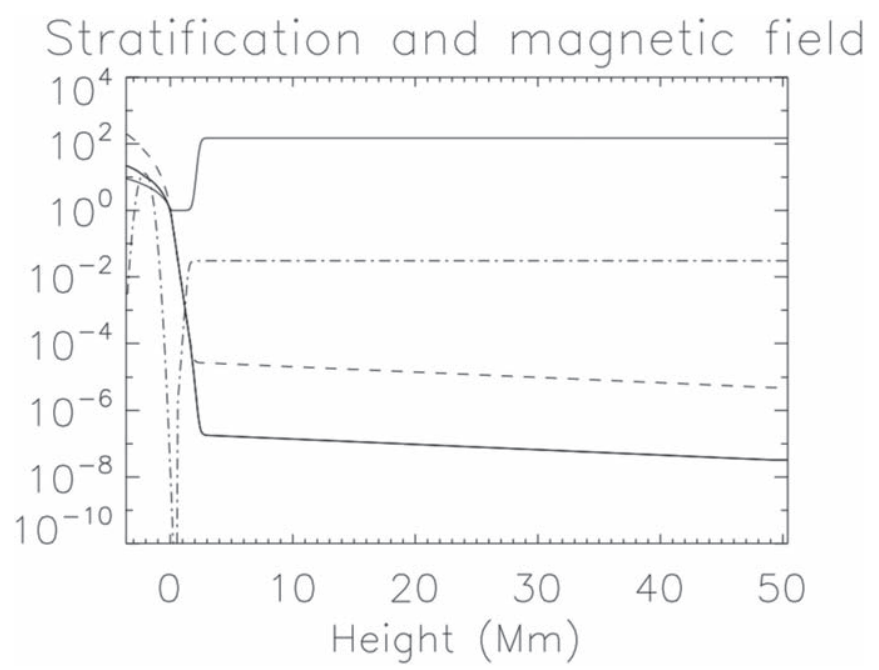

Figure 1. Initial stratification of the atmosphere and the magnetic field in dimensionless units: temperature (thin solid), gas pressure (dashed), density (thick solid), and magnetic field (dotted-dashed). The dimension units we use are temperature $T=5100 \mathrm{~K}$, pressure $P=7.16 \times 10^{3} \mathrm{erg} \mathrm{cm}^{-3}$, density $\rho=1.67 \times 10^{-7} \mathrm{~g} \mathrm{~cm}^{-3}$, and magnetic field strength $B=300 \mathrm{G}$.

$$
\boldsymbol{E}^{*}=\boldsymbol{E}-\frac{m_{0}}{q} u_{\|} \frac{\partial \hat{\boldsymbol{b}}}{\partial t},
$$

with $m_{0}$ and $q$ being the particle's rest-mass and charge, respectively, and $\mu$ is the magnetic moment,

$$
\mu=\frac{m_{0} u_{\perp}^{2}}{2 B}
$$

and $\gamma$ is the Lorentz factor

$$
\gamma=\frac{1}{\sqrt{1-(v / c)^{2}}}=\sqrt{1+\frac{u^{2}}{c^{2}}}
$$

The evolving velocity variable $u_{\|}$is the parallel component of the four-velocity $\boldsymbol{u}=\gamma \boldsymbol{v}(\boldsymbol{v}=d \boldsymbol{r} / d t)$, and $u_{\perp}$ is the perpendicular component of the latter. The electromagnetic fields of the MHD simulations are interpolated in three dimensions with local third-order polynomials (tri-cubic interpolation), which are continuous over the grid points in the components and the derivatives up to first and partially to second order. The parallel electric field is interpolated explicitly, i.e., it is not calculated in-between the grid points from the interpolated three Cartesian electric field components, because with this procedure we avoid effects of artificial particle energization. The temporal integration of the equations of motion is done with the Dormand-Prince scheme of the family of Runge-Kutta methods, with an adaptive time step.

Collisions, when taken into account, are implemented as a Monte Carlo method, i.e., we consider the collisions as a stochastic process and superpose it onto the deterministic motion, as outlined by Hamamatsu et al. (2007; see also Karney 1986).

\section{Statistical Properties of the Electric Field Activity}

\subsection{Overview over the MHD Snapshots}

With the focus of this study being on heating and acceleration in coronal active regions, we consider only the coronal part of the MHD simulation box. We consider two time instances (termed snapshots in the following) out of the MHD simulation: (i) snapshot 30 (at $30 \mathrm{~m}$ from the simulation start), which shows a clearly shaped standard jet as a result of reconnection between the emerging and pre-existing magnetic fieldlines, and (ii) snapshot 53 (at $53 \mathrm{~m}$ ), at which a magnetic flux rope has been formed within the emergence region, and it erupts to drive the emission of an explosive "blowout" jet. Note that the time interval between two MHD snapshots is $t_{0}=85.7 \mathrm{~s}$, whereas the integration time of the test particles is of the order of $1 \mathrm{~s}$, so there is no need for, e.g., interpolating in the time direction between two subsequent MHD snapshots, and we can consider one single snapshot and assume that it does not evolve over the kinetic timescale of interest. Figure 2 shows magnetic fieldlines and isocontours of the magnitude of the total electric field $|\boldsymbol{E}|$ (left panel, snapshot 30), and isocontours of the parallel electric field $E_{\|}$, including the sign (right panel, snapshot 30). Both the parallel and the total electric field are distributed along the main reconnection regions. These are the regions where current sheets are built up and the emerging field starts to reconnect with the pre-existing magnetic field. We find that the spatial distribution of the electric field reveals the formation of large-scale structures (i.e., the current sheets) and their tendency to break up into fragments, in different degrees though, with the parallel electric field clearly being more fragmented. Figure 3 shows the topology of the overall system at snapshot 53 of the MHD simulation. The magnetic fieldlines within the central emergence region are more twisted. This is due to the formation of a magnetic flux rope, owing to shearing and reconnection of fieldlines above the polarity inversion line of the emerging bipolar region. As the flux rope rises, it starts to reconnect with the ambient field. Eventually, it erupts, driving the onset of a helical blowout jet. The spatial distribution of the electric field reveals two main regions where the electric fields are strong. One region is above the flux rope (red isosurface) and it shows the location of a large-scale current sheet at the interface between the erupting flux rope and the ambient coronal field. The second region is underneath the flux rope (yellow isosurface), and it marks the location of the current sheet above the polarity inversion line where sheared fieldlines reconnect to form the flux rope.

\subsection{Statistics of the MHD Electric Field}

Figure 4 shows the histogram of the magnitude of the total electric field $|\boldsymbol{E}|$, the parallel $\left|E_{\|}\right|$, and the perpendicular $E_{\perp}$ components of the electric field, determined from all coronal grid points. They all show a power-law tail with a rollover at high values. The power-law index of the fit is -1.8 for the parallel electric field, and -2.4 for the total and perpendicular electric field in case of snapshot 30, and for snapshot 53 the values are similar, just that the total and perpendicular electric field attain larger values. In any case, the parallel electric field is two orders of magnitude smaller than the total electric field, which thus basically coincides with the perpendicular electric field. Also, the parallel electric field shows a much more extended power-law tail than the perpendicular and the total one. We thus conclude that power-law-shaped distributions are inherent to the electric field and its components. Similar results have also been found in MHD simulations of a decaying current sheet (see Figure 2 in Onofri et al. 2006) and of a twisted coronal loop (see Figure 2(b) in Turkmani et al. 2006). 

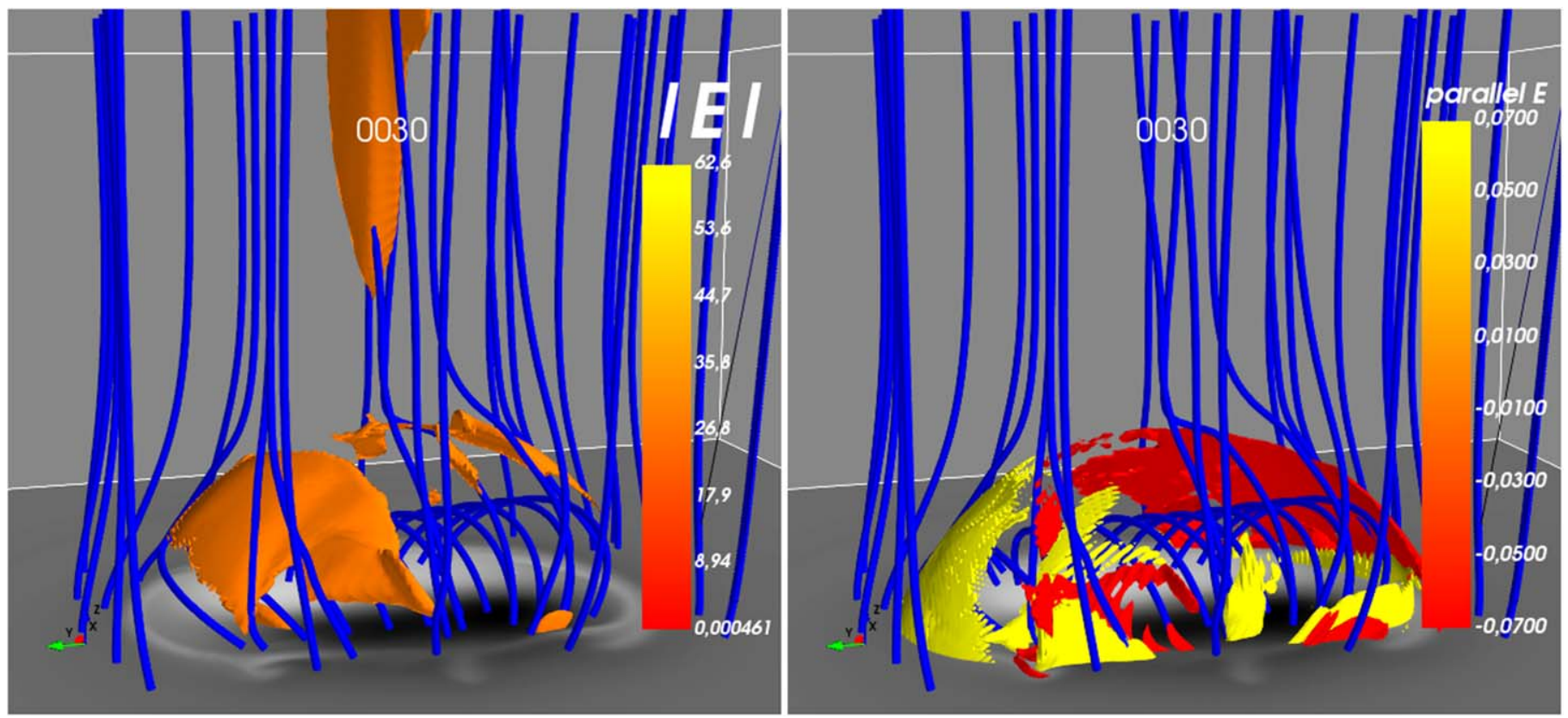

Figure 2. Results from the MHD simulations; a close-up of the coronal part, snapshot 30. The left panel shows a visualization of selected magnetic fieldlines (blue) together with an isocontour plot of the total electric field (orange 3D isosurfaces). The vertically oriented isosurface (orange) is aligned with the direction of the reconnected fieldlines and it indicates the emission of the standard jet. The horizontal $x-y$-plane shows the photospheric component $B_{z}$ as a $2 \mathrm{D}$ filled contour plot. The electric field is in physical units $\left[\mathrm{V} \mathrm{m}^{-1}\right]$. In the right panel, isocontours of the parallel electric field are shown, indicating the fragmentation of the current sheet(s) at the interface between the interacting fields.

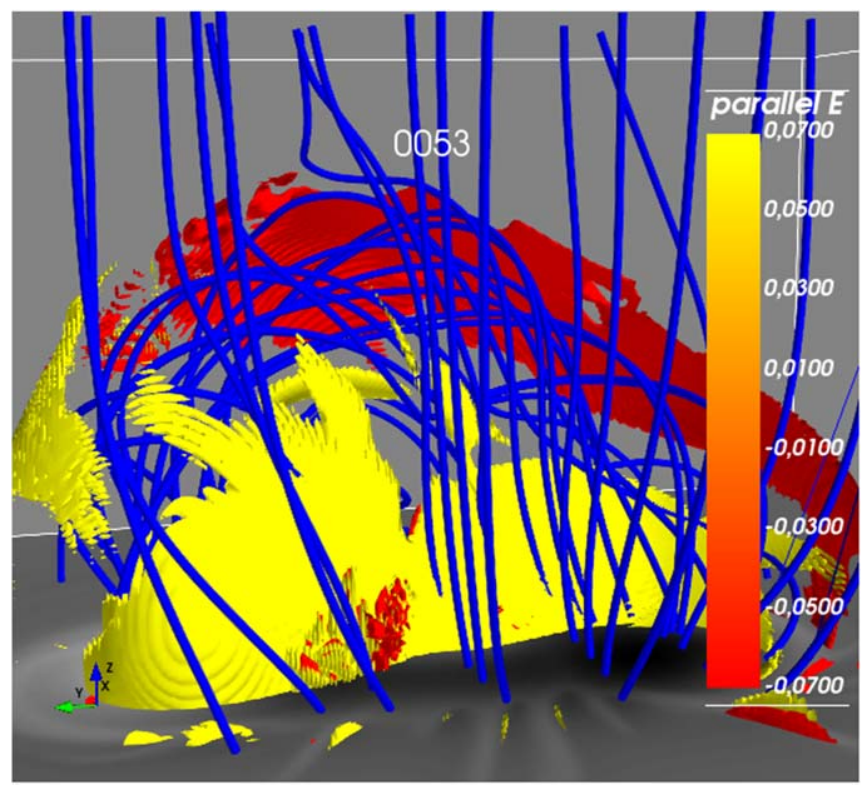

Figure 3. Same as Figure 3, for the parallel electric field, and for snapshot 53. The eruption of the twisted magnetic field structure (flux rope) drives the emission of a blowout jet.

The Dreicer field is given as

$$
E_{D}=7 \cdot 10^{-4} \frac{n}{10^{9} \mathrm{~cm}^{-3}}\left(\frac{T}{10^{7} \mathrm{~K}}\right)^{-1} \frac{\ln \Lambda}{23.2} \mathrm{~V} \mathrm{~m}^{-1}
$$

(e.g., Holman 1985), so for typical coronal values used in the MHD simulations, $n=1.0 \times 10^{10} \mathrm{~cm}^{-3}, T=7.6 \times 10^{5} \mathrm{~K}$, $\ln \Lambda=23.2$, we have the mean value

$$
\left\langle E_{D}\right\rangle=5.0 \times 10^{-4} \mathrm{~V} \mathrm{~m}^{-1},
$$

with maximum value $2 \times 10^{-2} \mathrm{~V} \mathrm{~m}^{-1}$ and minimum value $2 \times 10^{-6} \mathrm{~V} \mathrm{~m}^{-1}$. It thus follows from Figure 4 that the perpendicular electric field is highly super-Dreicer almost everywhere, whereas the parallel one attains highly superDreicer values only at a fraction of the grid points. The threshold \pm 0.07 chosen for the isocontours of the parallel electric field in Figure 2 (right panel) and Figure 3 corresponds to $140\left\langle E_{D}\right\rangle$, implying that the parallel electric field is highly super-Dreicer in a wider region enclosing the main locations of reconnection and the outflow region.

\subsection{Statistics of the MHD Energies}

Figure 5 shows the kinetic energy distribution of the $\mathrm{E}$ cross B velocity, $\frac{1}{2} m_{e}\left(\boldsymbol{E} \times \boldsymbol{B} / B^{2}\right)^{2}$, and the MHD flow velocity, $\frac{1}{2} m_{e} \boldsymbol{V}^{2}$, as calculated from all the coronal grid points. For snapshot 30, the two distributions are very similar in shapeexcept for the lowest energies. They show a power-law tail with index -1.61 , and the highest energy reached is $0.1 \mathrm{keV}$. Also shown in the figure is the MHD thermal energy distribution, $\frac{3}{2} k_{\mathrm{B}} T$, which reaches a maximum value of $0.5 \mathrm{keV}$, has a power-law decay with index -0.92 , and exhibits a clear peak near $0.1 \mathrm{keV}$. In the case of snapshot 53 , the situation is rather similar, just that roughly one order of magnitude larger energy values are reached.

Figure 6 shows the distribution of the energy contained in the parallel electric field,

$$
W_{E_{\|}}=\frac{1}{2} \epsilon E_{\|}^{2}
$$

(with the permittivity $\epsilon \approx \epsilon_{0}$ ). For both snapshots considered, the distribution has a double power-law form, extending over many decades, with index -0.50 in the low-energy part and 

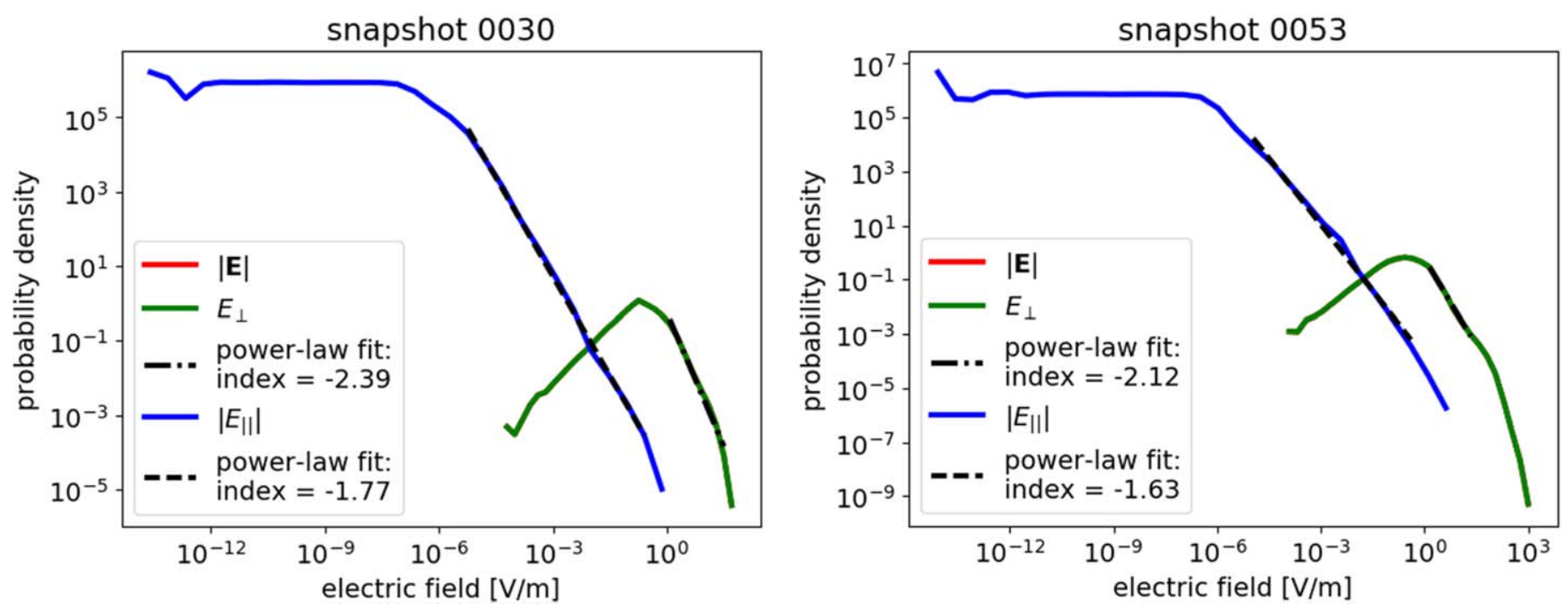

Figure 4. MHD simulations: snapshots 30 and 53, coronal part only, showing the distribution of the electric field from all coronal grid points, for the magnitude of the total electric field, the perpendicular component (they practically coincide), and the parallel component, respectively. The electric field is in units [V $\mathrm{m}^{-1}$ ], and the mean Dreicer field is $4.6 \times 10^{-4} \mathrm{~V} \mathrm{~m}^{-1}$.
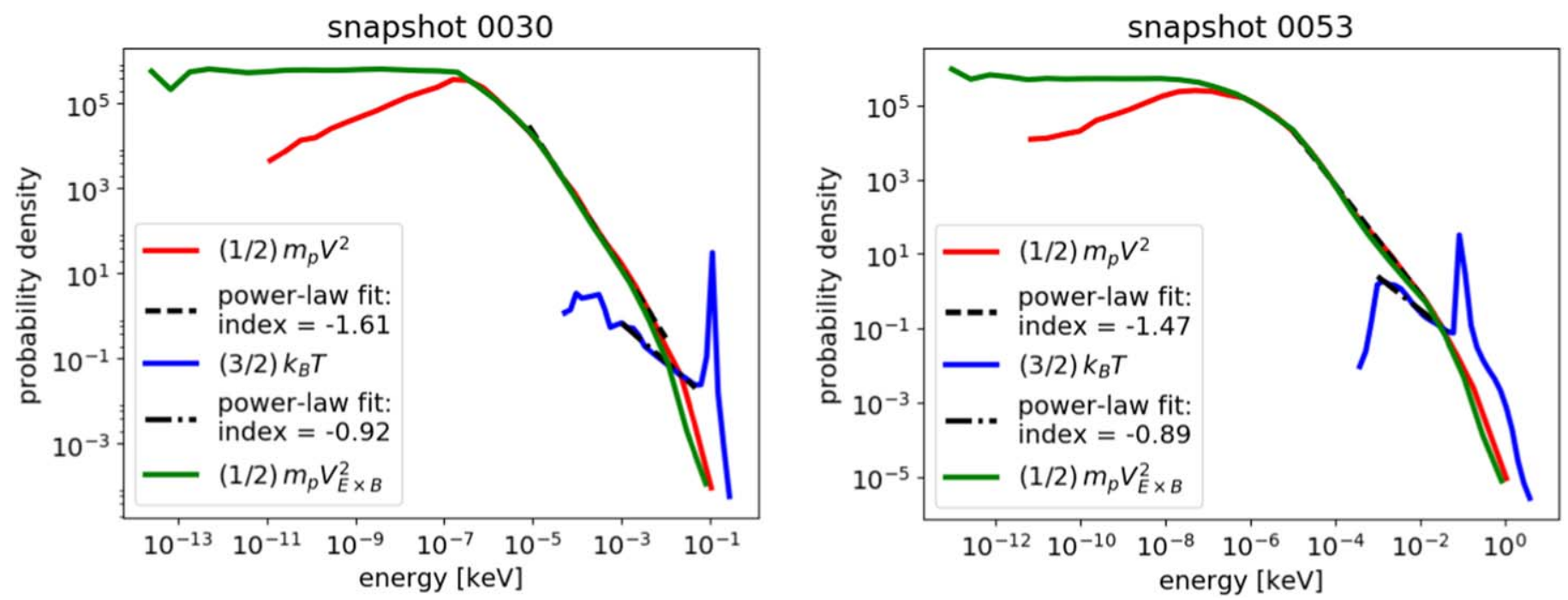

Figure 5. MHD simulations: snapshots 30 and 53, coronal part only, showing the distribution of the kinetic energy $(1 / 2) m_{P} V_{E \times B}^{2}$ of the E cross B drift velocity $V_{E \times B}$, of the kinetic energy $(1 / 2) m_{P} V^{2}$ of the MHD flow velocity $V$, and of the thermal energy $(3 / 2) k_{\mathrm{B}} T$, as determined from all the coronal grid points.

with index -1.4 and -1.3 , respectively, in the high-energy part, and where the maximum energy reached is about $10 \mathrm{MeV}$ for snapshot 30, and almost $100 \mathrm{MeV}$ for snapshot 53.

It thus follows that all the MHD energies of interest exhibit power-law distributions, with relatively low maximum values, of the order of the thermal energy (about $100 \mathrm{eV}$ ), with the exception of the energy contained in the parallel electric field that reaches the $\mathrm{MeV}$ regime. In the case of an emitted blowout jet, we find that the MHD energies are generally one order of magnitude larger than those at the standard jet.

\subsection{Spatial Structure of the Parallel Electric Field}

We now investigate the spatial structure of the parallel electric field, applying cluster analysis and calculating its fractal dimension.

\subsubsection{Cluster Analysis}

We consider the magnitude of the parallel electric field $\left|E_{\|}\right|$at all the coronal grid points, and we apply a threshold below which $\mid E_{\|}$is set to zero. For the threshold we use the same value of 0.07 as for the isocontours of $E_{\|}$in Figures 2 and 3.

We define a cluster as a set of grid points with (a) an abovethreshold value of $\left|E_{\|}\right|$at all the grid points belonging to the cluster, and (b) the cluster's grid points are connected through their nearest neighborhoods in 3D Cartesian coordinates. It follows that a cluster is surrounded by grid points with belowthreshold $\left|E_{\|}\right|$. Defined in this sense, the set of all clusters is related to the (above-threshold) isocontours in Figures 2 and 3; however, the cluster analysis splits the isocontours into parts, the clusters, which are not connected through the nearest neighborhoods of the grid points. Each cluster is numbered uniquely, and the grid points belonging to it are marked correspondingly. 

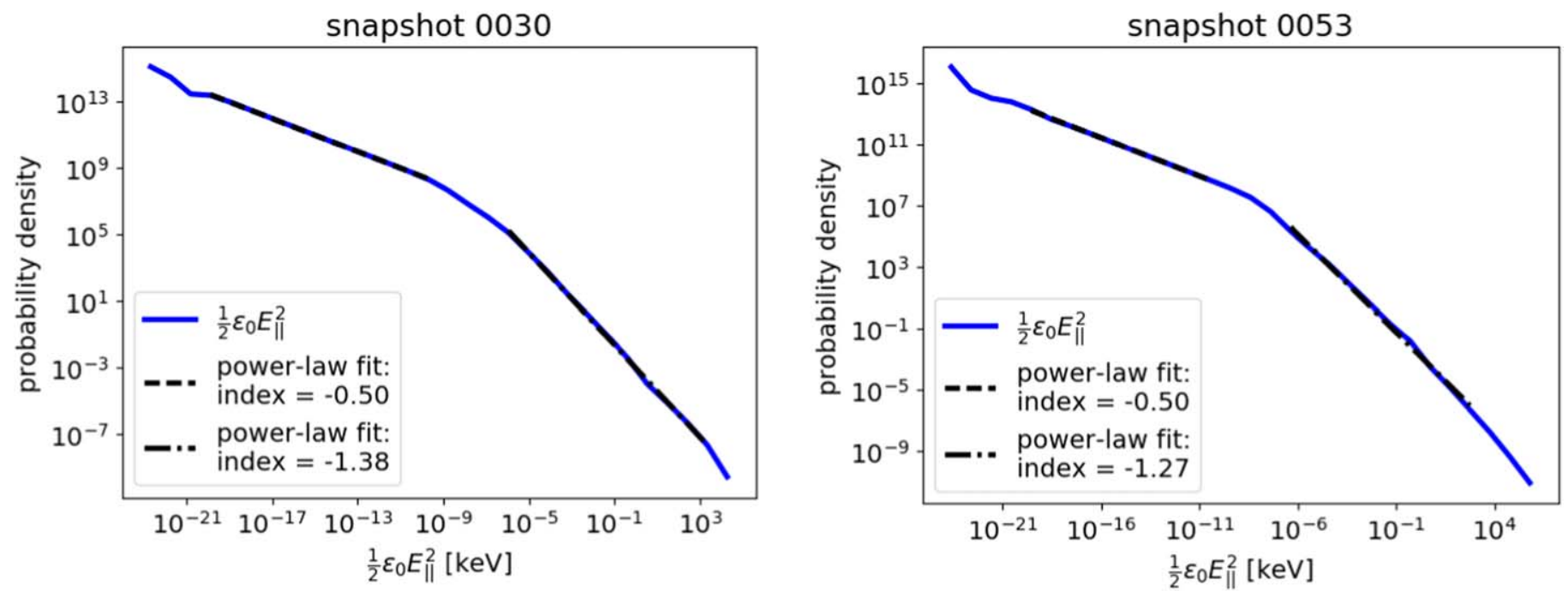

Figure 6. MHD simulations: snapshots 30 and 53 , coronal part only, showing the distribution of the parallel electric field energy density $\frac{1}{2} \epsilon E_{\|}^{2}$, as determined from all the coronal grid points.
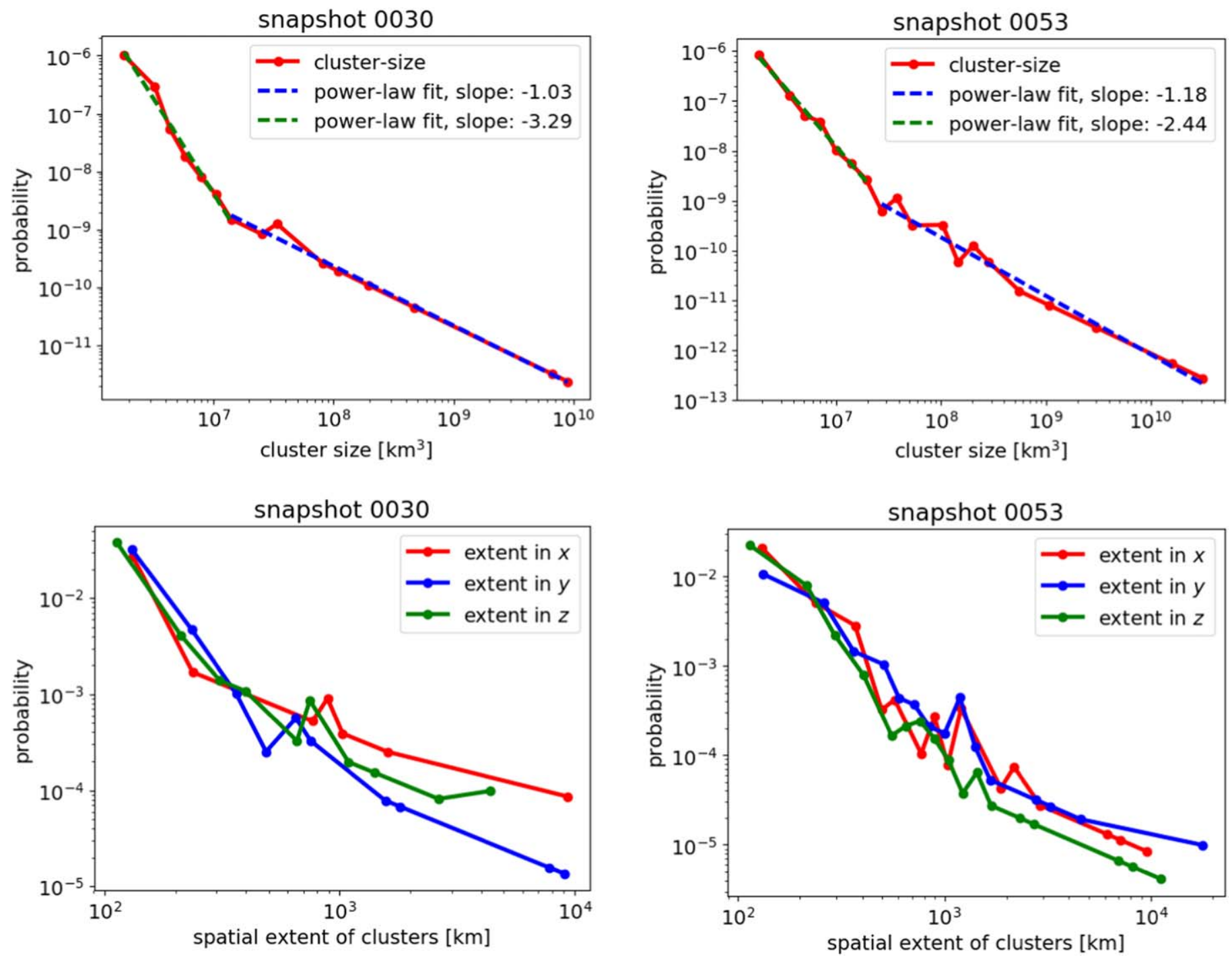

Figure 7. MHD simulations: snapshots 30 and 53, coronal part. Top: cluster size distribution. Bottom: distributions of spatial extent in the $x, y$, and $z$ directions. 

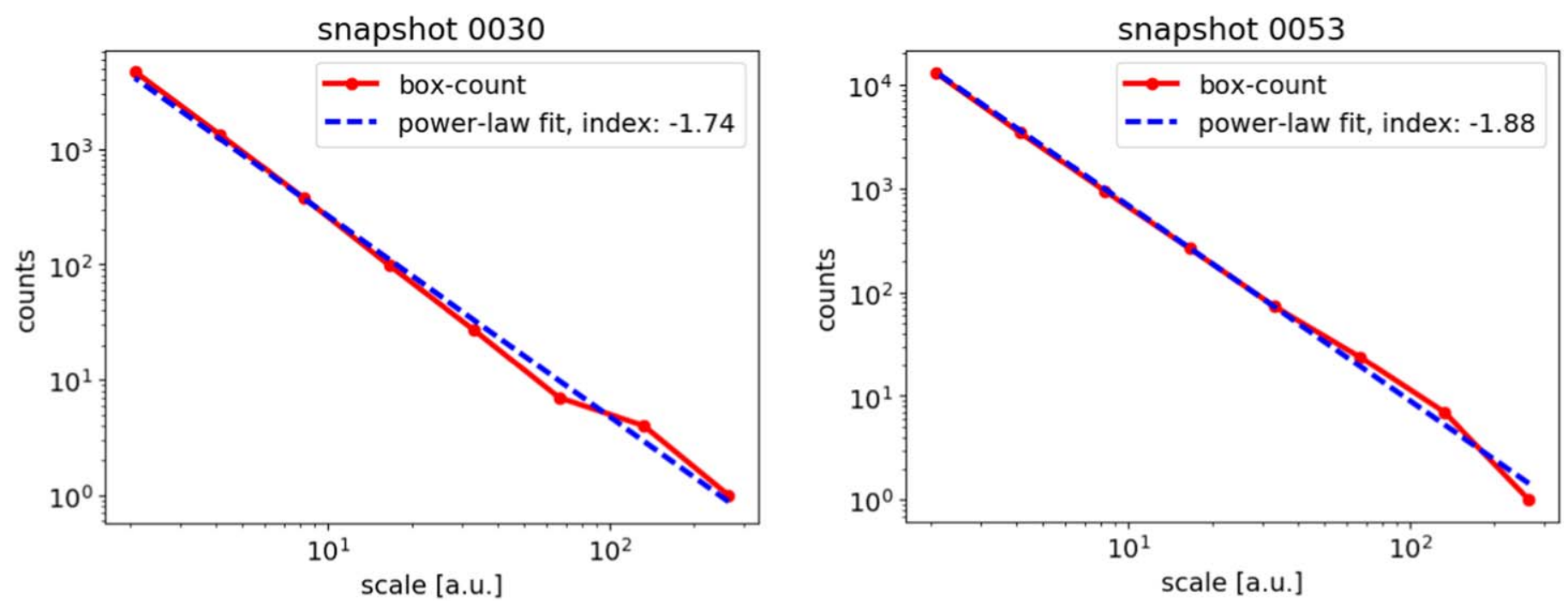

Figure 8. Fractal dimension of the parallel electric field: scaling of the 3D box-counting algorithm, for snapshots 30 and 53.

For snapshot 30, we find that there are 162 clusters, and 2 of them are very dominant in spatial extent, one corresponding to the positive and one to the negative extended parallel electric field region in Figure 2.

For each cluster, we determine the cluster size as the number of grid points belonging to the cluster times the elementary grid volume $\Delta x \Delta y, \Delta z$. The distribution of cluster sizes is shown in Figure 7, there is a double power law for both snapshots, with a steep decay at small sizes with power-law index 3.3 and 2.4 , and a flatter power-law scaling at the larger sizes, with index 1.0 and 1.2 , for snapshots 30 and 53, respectively.

Figure 7 also shows the distribution of the linear cluster extents in the $x, y$, and $z$ directions. There again is a vague double power-law scaling, with the sizes being larger at the blowout jet than at the standard jet.

\subsubsection{Fractal Dimension}

Using the same data employed in the cluster analysis (the magnitude of the parallel electric field $\left|E_{\|}\right|$at all the coronal grid points, set to zero when below the threshold value of 0.07 ), we apply a standard 3D box-counting method in order to determine the fractal dimension $D_{F}$ of the region with above-threshold parallel electric field. Figure 8 shows the scaling of the box counts with the box scale, where there is a clear power-law scaling in the entire range, whose index, per definition of the box-counting method, equals the fractal dimension, so we find $D_{F}=1.7$ for snapshot 30 and $D_{F}=1.9$ for snapshot 30 .

The regions of high parallel electric field can thus be interpreted as thinned out 2D sheets, as it also corresponds to the visual impressions that are given by Figures 2 and 3, and whereby the "filling-factor" is higher at the blowout jet compared to the time when the standard jet is emitted.

After all, the spatial structure of the regions of strong parallel electric field can be characterized as fragmented and fractal, with the various size distributions exhibiting double power-law scalings.

\section{The Evolution of the Test Particles in the Vicinity of the Standard Jet}

We first consider the energization of particles at the standard jet, snapshot 30 . The electric and magnetic fields from the MHD simulations are denormalized to SI units and are not

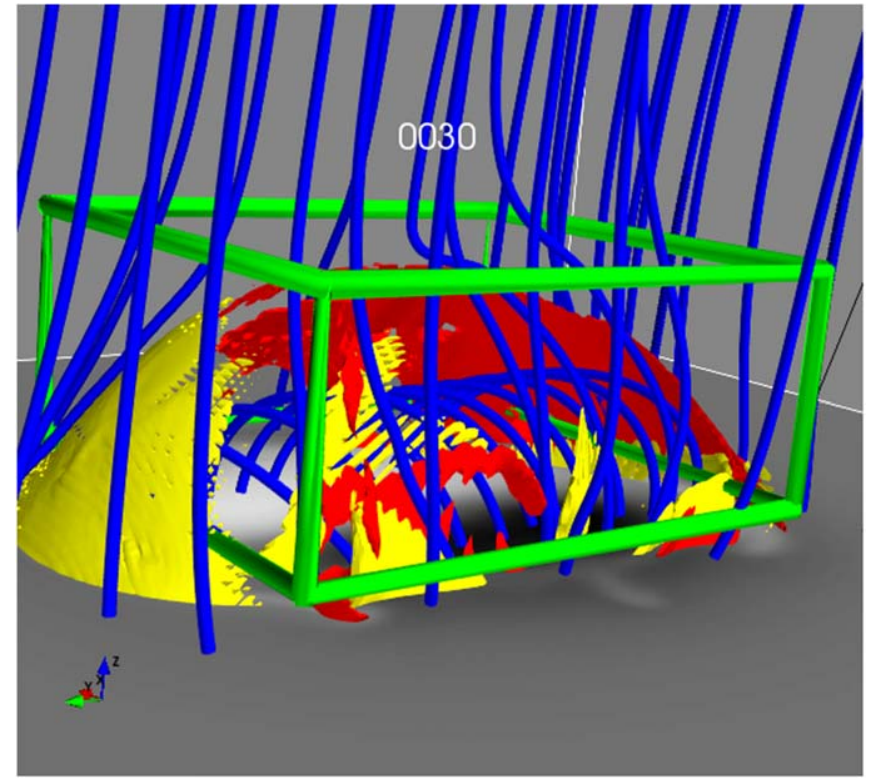

Figure 9. MHD simulations, snapshot 30 , zoomed into the coronal part: magnetic fieldlines are shown (blue), together with an isocontour plot of the parallel electric field (red to yellow 3D surfaces), for the same two thresholds indicated by the color bar in Figure 2. At the bottom $x-y$ plane, the photospheric component $B_{z}$ is shown as a $2 \mathrm{D}$ filled contour plot. The region in which the spatial initial conditions are chosen is outlined by a green cube.

scaled further. Electrons are considered test particles, and, if not mentioned otherwise, the standard integration time is $0.1 \mathrm{~s}$, and 100,000 particles are traced, in any case, using the relativistic guiding-center approximation to the equations of motion; see Section 2.2. The initial spatial position is uniform random in the region around the main reconnection region, as outlined by the green cube in Figure 9, which basically contains the entire current sheet with all its fragments. The initial velocity is random, with a Maxwellian distribution (i.e., a Gaussian distribution of the velocity components), with temperature $\approx 9 \times 10^{5} \mathrm{~K}$. For each simulation, a set of 100 monitoring times has been predefined, including the final time, at which the velocities and positions of the particles are monitored for the purpose of a statistical analysis to be done at 

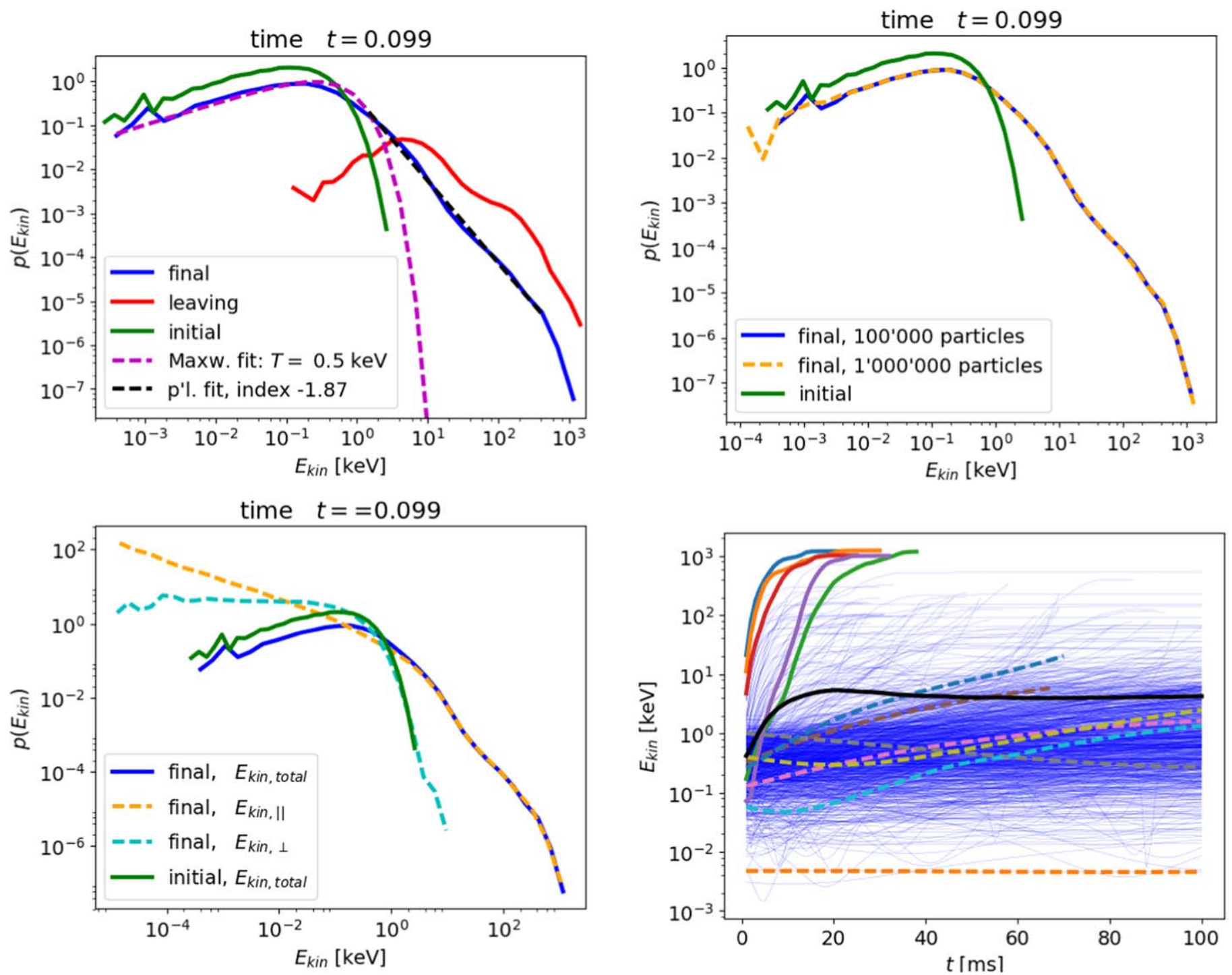

Figure 10. Snapshot 30: kinetic energy distribution of electrons after $\approx 0.1 \mathrm{~s}$ (top left), without collisions, together with a fit at the low-energy, Maxwellian part and the high-energy, power-law part, the initial distribution, and the distribution of the leaving particles (for every particle at the time it leaves). Top right: comparison of the kinetic energy distributions from simulations with 100,000 and 1,000,000 test particles, respectively. Bottom left: comparison of the total kinetic energy, the parallel, and the perpendicular kinetic energy at $0.1 \mathrm{~s}$. Bottom right: kinetic energy of particles as a function of time (thin blue lines), with a few trajectories marked with bold lines (solid at high energies and dashed at low energies), together with the mean energy (solid black).

equal times for all the particles. A separate track is kept of the particles that leave before the final time.

\subsection{Acceleration}

The top left panel of Figure 10 shows the distribution of the kinetic energies of the particles after $0.1 \mathrm{~s}$, together with the initial distribution and the distribution of the leaving particles (as collected at the times the individual particles leave). The final energy distribution has a Maxwellian shape at low energies, and exhibits a slightly modulated power-law tail. The maximum energy reached is about $1 \mathrm{MeV}$, and a power-law fit to the tail of the kinetic energy distribution yields an index of about -1.87 .

$13 \%$ of the 100,000 particle that are traced have left after $0.1 \mathrm{~s}$, and they have energies in the same range than those that stay inside, with a modulated power-law tail that is steeper though, with an index -2.98 at the highest energies (the fit is not shown).

In the bottom left panel of Figure 10 we separately show the final total, parallel, and perpendicular kinetic energy at $0.1 \mathrm{~s}$.
Obviously, the power-law tail in the total kinetic energy is inherited from the parallel kinetic energy, there is essentially no energization in the perpendicular direction, as expected from Figure 5, with the energy in the E cross B velocity having a maximum value of only $0.1 \mathrm{keV}$. Thus, an important conclusion is that acceleration is acting exclusively in the parallel direction.

In order to check how reliable the statistical sample of 100,000 particles is, we performed a simulation with 10 times more particles $(1,000,000)$, and from the top right panel of Figure 10, it can be seen that the kinetic energy distribution does not change when using a substantially higher number of particles; in particular, the maximum energy reached also remains unchanged. We thus will use throughout 100,000 particles in the simulations presented.

Figure 10, bottom right, shows the kinetic energy of the particles as a function of time, with a few low-energy and a few high-energy particles marked, together with the mean energy. The high-energy particles tend to reach their final energy in a 

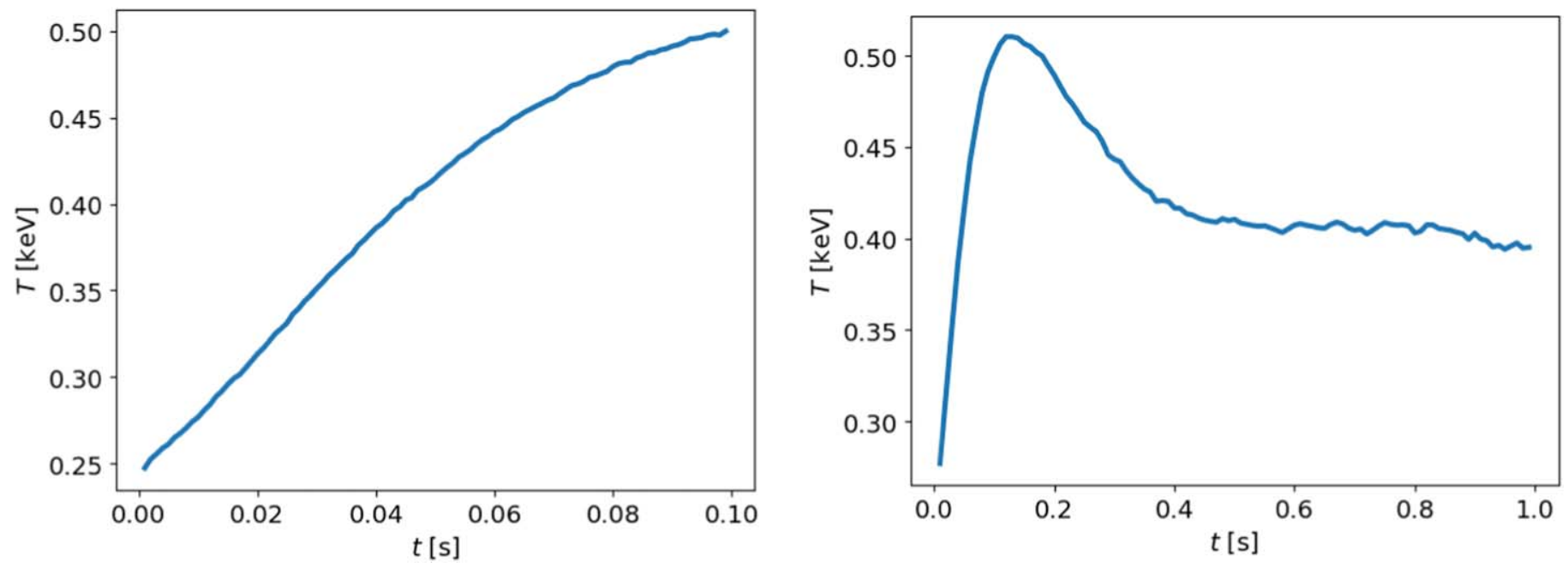

Figure 11. Snapshot 30: temperature as a function of time, as determined through the fit of a Maxwellian in the low-energy range of the kinetic energy distribution.

fast and single step, with a timescale of the order of some tens of milliseconds. The low-energy particles evolve on a much slower timescale, of the order of seconds, during which the energy gradually increases; in particular, they show a slow drift motion rather than a classical random walk.

\subsection{Heating}

For the particles that stay inside, the Maxwellian shape of the energy distribution is well preserved at low energies, and there is heating from the initial $0.24 \mathrm{keV}$ to $0.50 \mathrm{keV}$ after $0.1 \mathrm{~s}$, as the Maxwellian fit in the top left panel of Figure 10 reveals. Figure 11 shows the temperature as a function of time, as estimated by Maxwellian fits to the kinetic energy distribution in the low-energy part. The temperature increases linearly with time until $0.05 \mathrm{~s}$ and then starts to turn over, reaches a peak value of $0.50 \mathrm{keV}$ at $0.1 \mathrm{~s}$, and finally the heating process saturates for times larger than roughly $0.5 \mathrm{~s}$ with a temperature of $0.40 \mathrm{keV}$. The decrease from 0.5 to $0.4 \mathrm{keV}$ may be attributed to the loss of a fraction of the higher-energy bulk particles.

The energy distribution of the leaving particles shows a functional form at low energies (between 0.1 and $10 \mathrm{keV}$ ) that is reminiscent of a Maxwellian, and a respective fit reveals a temperature of about $13.3 \mathrm{keV}$ (see Figure 10, top left, the fit itself is not shown). Although the statistics is not very good, we can interpret these particles as belonging to a super-hot population. Note, however, that the energies are monitored at different times for each particle, so the distribution is asynchronous.

\subsection{Longer and Shorter Times}

Considering times longer than $0.1 \mathrm{~s}$, we find that at a final time of $1.0 \mathrm{~s}, 57 \%$ of the particles have left the system. The kinetic energy distribution of the particles is shown in Figure 12, bottom right. The tail of the distribution for the particles that remain inside now has a clear power-law part only at the highest energies, with an index -1.0 , much smaller than the one at $0.1 \mathrm{~s}$. The intermediate-to-high-energy part does not show a clear scaling anymore, however, and the statistics has become poor due to the large number of particles that have left. Note that the highest energy reached, about $20 \mathrm{MeV}$, is much larger in comparison to the time $0.1 \mathrm{~s}$. At low energies, the particles are heated to a temperature of $0.40 \mathrm{keV}$, which is below the peak temperature reached at $0.1 \mathrm{~s}$.

The leaving particles have a modulated power-law tail, with an index -2.73 at the highest energies, which is close to the index seen at $0.1 \mathrm{~s}$, and the low-energy part is now closer to a Maxwellian shape, with a temperature of $7.5 \mathrm{keV}$, about onehalf of the temperature at $0.1 \mathrm{~s}$ (both fits are not shown in the figure).

Considering times shorter than $0.1 \mathrm{~s}$, we find that at $t \approx 0.01 \mathrm{~s}$ a power-law tail has already been formed with index -1.32 (Figure 12 top left), and at $t \approx 0.02 \mathrm{~s}$ a double power-law appears with index -1.82 in the high-energy part (Figure 12 top right). The power-law tail thus gets steeper in the initial phase, and then flattens for larger times.

\subsection{The Effect of Collisions}

We consider collisions with background electrons of the same temperature as the initial temperature of the test particles (see Section 2.2). As Figure 13 shows, collisions play a role at low energies only; as expected, they reduce the efficiency of the heating process, cooling down the electrons toward their initial temperature; the temperature reached at $0.1 \mathrm{~s}$ with collisions is $0.38 \mathrm{keV}$, compared to $0.50 \mathrm{keV}$ in the case without collisions. The cooling down of the test particles corresponds to a heating of the background population, which is not taken into account in our modeling approach.

\subsection{Particle Orbits}

The left panel of Figure 14 shows particle orbits for 40 randomly chosen particles. The particle energy is indicated by the color of the orbits. Being randomly chosen, the particles mainly belong to the population that is heated or moderately accelerated, and there is no discernible pattern revealing any preferences.

The right panel of Figure 14 shows the orbits of the 40 most energetic particles, and now there is clearly a preference in the initial conditions; there are two regions from where the energetic particles originate, one close to the region of strong positive parallel electric field, and one close to the region of strong negative parallel electric field. Also, most particles move some distance along the region of high electric field and pass through it at some point, whereby their energy increases 

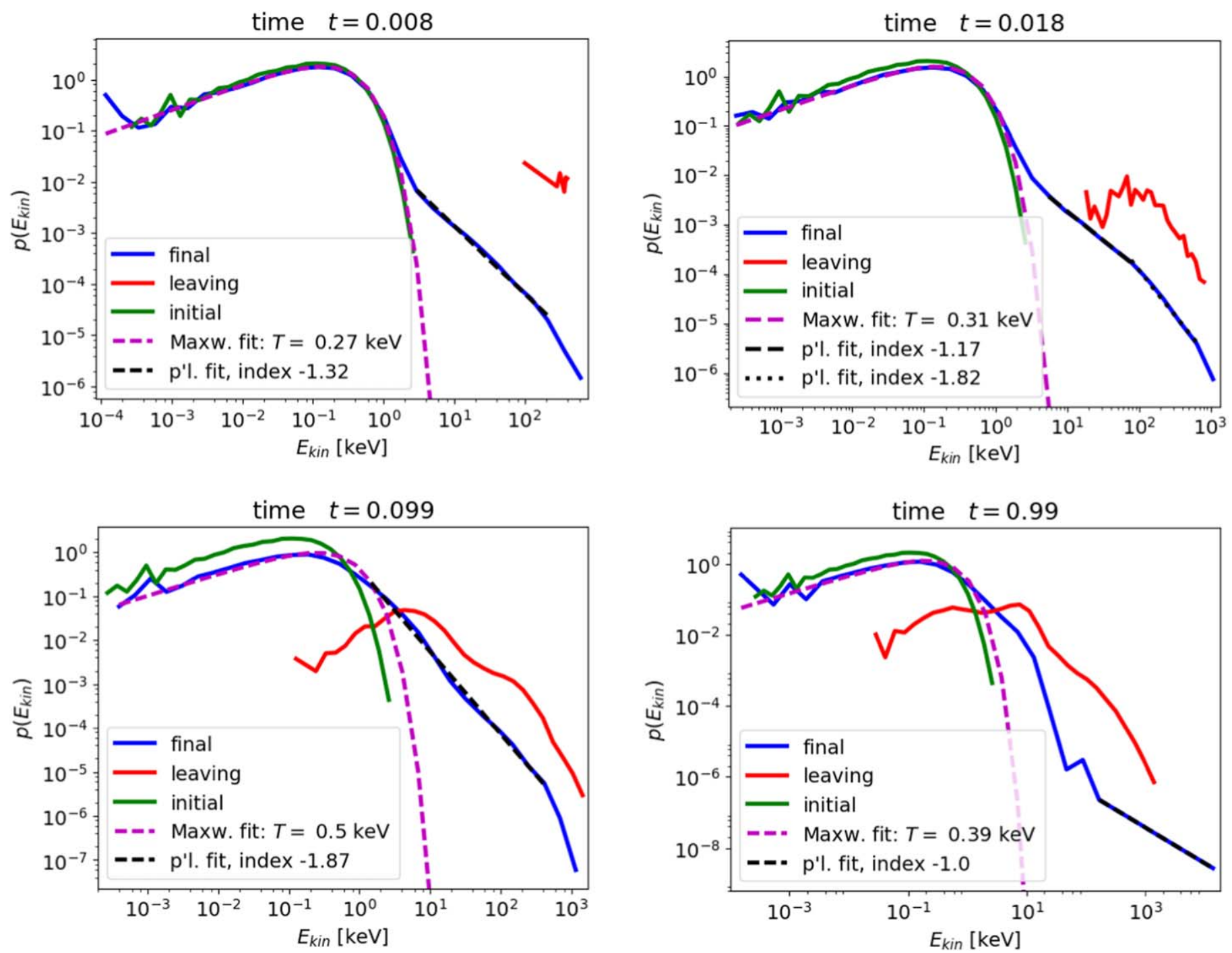

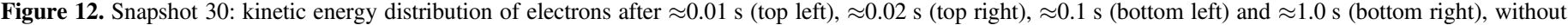

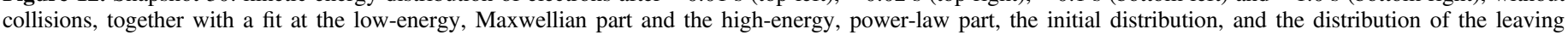
particles (for every particle at the time it leaves).

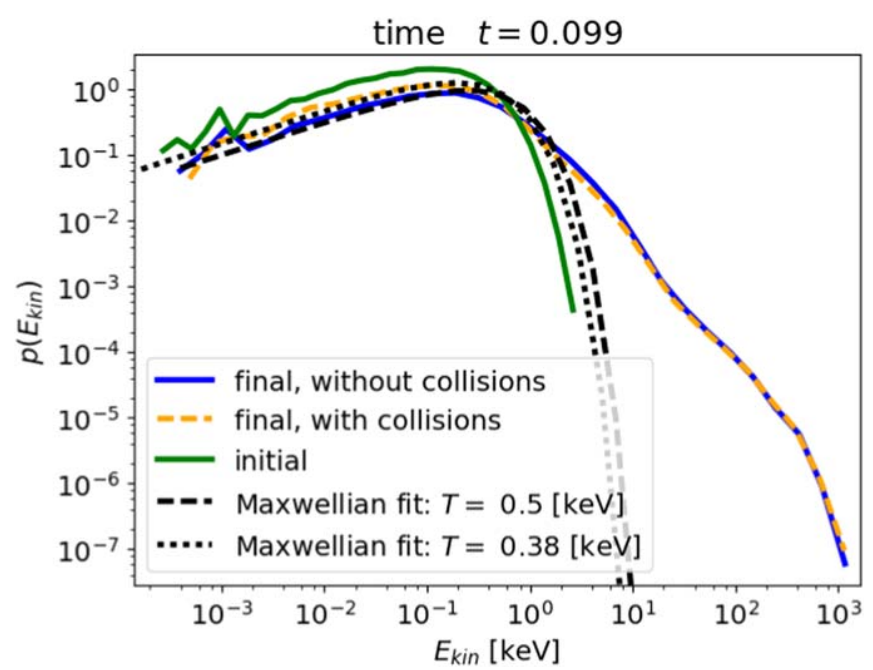

Figure 13. Snapshot 30: kinetic energy distribution of electrons after $0.1 \mathrm{~s}$, without collisions (blue) and with collisions (orange), together with the initial distribution (green). strongly, and after which they leave the simulation cube, mostly, but not entirely, through the bottom plane, toward the photosphere. The energetic particles thus undergo just one acceleration event, in accordance with the picture given in the bottom right panel of Figure 10 and hence the acceleration process is of a single and not a multiple nature.

\section{Particle Dynamics During the Blowout Jet Emission}

We now turn to snapshot 53, at which a well developed blowout jet has been formed. Figure 15 shows the magnetic configuration, the structure of the parallel electric field, and the region from which the initial conditions are chosen. Figure 16 shows the kinetic energy distribution at $t \approx 0.1 \mathrm{~s}$, there is heating to a temperature of $0.44 \mathrm{keV}$ at low energies, and there is also acceleration, with the power-law tail having an index -1.92 . Both the temperature and the power-law index are close to the values found for the case of the standard jet, but now the highest energy reached $(2 \mathrm{GeV})$ is twice as large.

The leaving particles show a clear power-law tail with index -1.8 , and they are heated to a temperature of $17.3 \mathrm{keV}$. The 

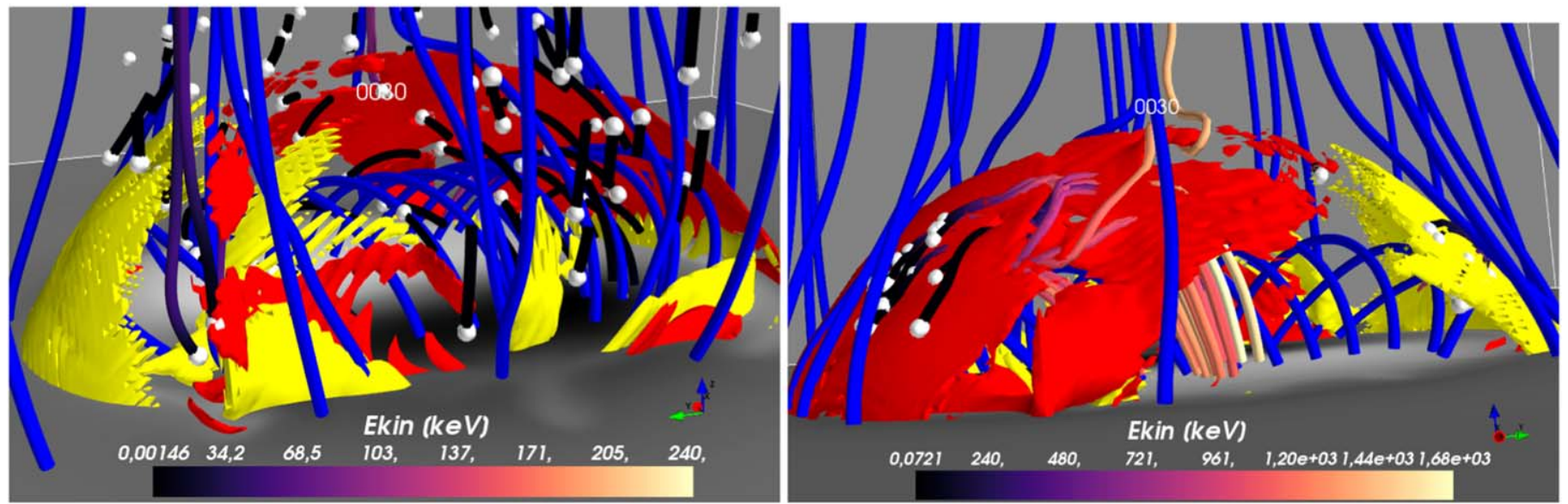

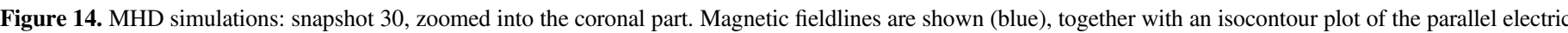

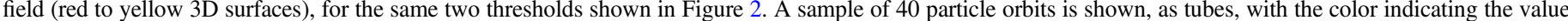

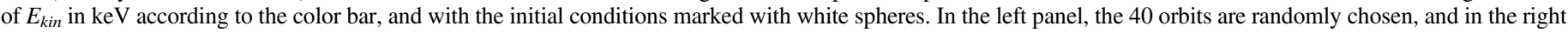
panel the orbits of the 40 most energetic particles are shown.

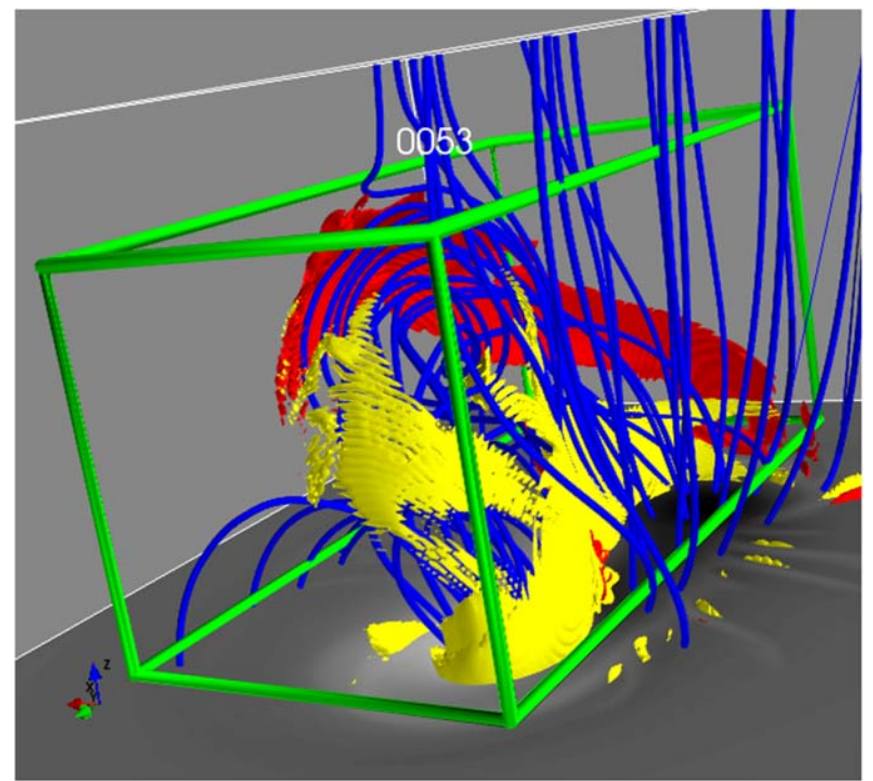

Figure 15. MHD simulations: snapshot 53, zoomed into the coronal part. Magnetic fieldlines are shown (blue), together with an isocontour plot of the parallel electric field (red to yellow 3D surfaces), for the same two thresholds shown in Figure 3. The green cube outlines the region from which the initial conditions are chosen.

temperature thus is close to the one reached at the standard jet; the power-law tail, though, is much flatter now.

The right panel of Figure 16 shows the kinetic energy of the particles as a function of time. Similar to the case of the standard jet, the low-energy particles drift slowly upward in energy, while the high-energy particles undergo basically one acceleration event (one-step or single acceleration).

The above results indicate that the particle energetics on the kinetic level are very similar in the standard and the blowout jet cases.

\section{Nature of Transport in Energy Space}

We now turn to the question of the nature of transport in energy space, in view of the results reported in the previous sections that are based on the test-particle approach.

\subsection{The Classical Fokker-Planck Equation Approach}

A first and classical candidate for a statistical transport model is the Fokker-Planck equation, which in energy space is written as

$$
\frac{\partial f}{\partial t}=\frac{\partial}{\partial E}\left[\frac{\partial(D f)}{\partial E}-F f\right]-\frac{f}{\tau_{\mathrm{esc}}},
$$

with $f$ being the probability density function of the kinetic energy, $D$ being the diffusion, $F$ being the convection coefficient, and $\tau_{\text {esc }}$ is the escape time. In this approach, the basic step is the determination of the two transport coefficients, $D$ and $F$, which we derive here from the test-particle simulation data.

In the following, we denote with $E_{k i n, j}\left(t_{k}\right)$ the kinetic energy of the particle with index $j$ at predefined, equally spaced, monitoring times $t_{k}\left(k=1, \ldots, 100, t_{100}\right.$ is the final time). In order to determine the energy-dependent and time-dependent transport coefficients from the test-particle simulation data, we follow Ragwitz \& Kantz (2001), whose definition is based on the time-dependent energy differences:

$$
\epsilon_{j}\left(t_{k}\right):=E_{k i n, j}\left(t_{k+h}\right)-E_{k i n, j}\left(t_{k}\right),
$$

with $h$ as the lag index, and usually we set $h=1$.

An estimate of the energy dependence of the transport coefficients, for a given time $t_{k}$, is made by first prescribing bins along the $E_{k i n}$-axis, with midpoints $E_{i}(i=1, \ldots, n)$, and then considering $E_{k i n, j}\left(t_{k+h}\right)-E_{k i n, j}\left(t_{k}\right)$ a function of $E_{i}$ if $E_{k i n, j}\left(t_{k}\right)$ lies in the bin $i$. The functional form of the transport coefficients, defined as

$$
\begin{aligned}
D\left(t_{k}, E_{i}\right)= & \frac{1}{2\left(t_{k+h}-t_{k}\right)}\left\langle\left( E_{k i n, j}\left(t_{k+h}\right)\right.\right. \\
& \left.\left.-E_{k i n, j}\left(t_{k}\right)\right)^{2}\right\rangle_{j}\left(t_{k}, E_{i}\right),
\end{aligned}
$$

and

$$
\begin{aligned}
F\left(t_{k}, E_{i}\right)= & \frac{1}{\left(t_{k+h}-t_{k}\right)}\left\langle E_{k i n, j}\left(t_{k+h}\right)\right. \\
& \left.-E_{k i n, j}\left(t_{k}\right)\right\rangle_{j}\left(t_{k}, E_{i}\right)
\end{aligned}
$$



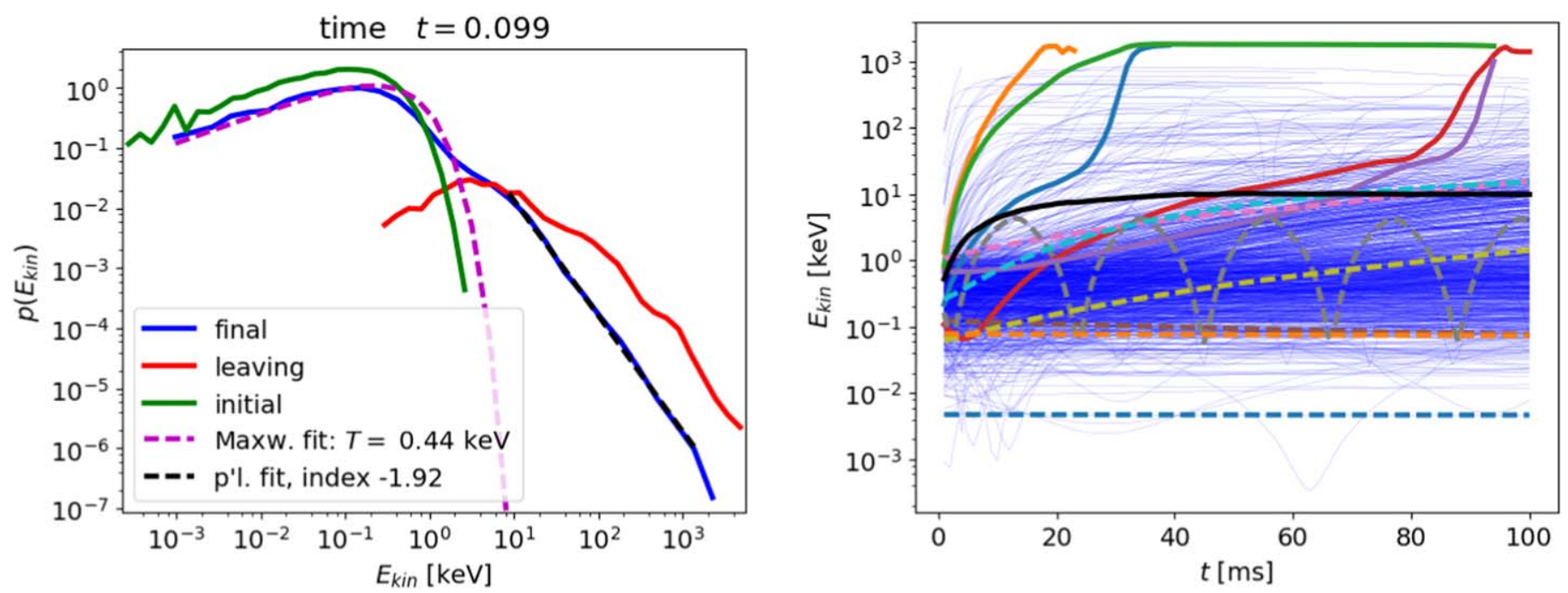

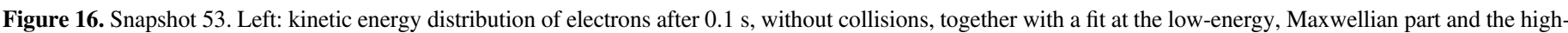

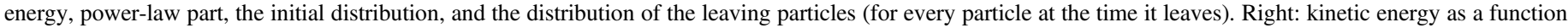

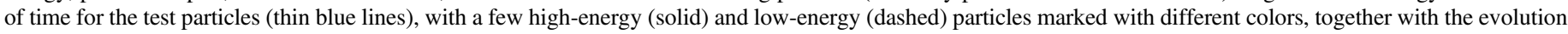
of the mean value (solid black).
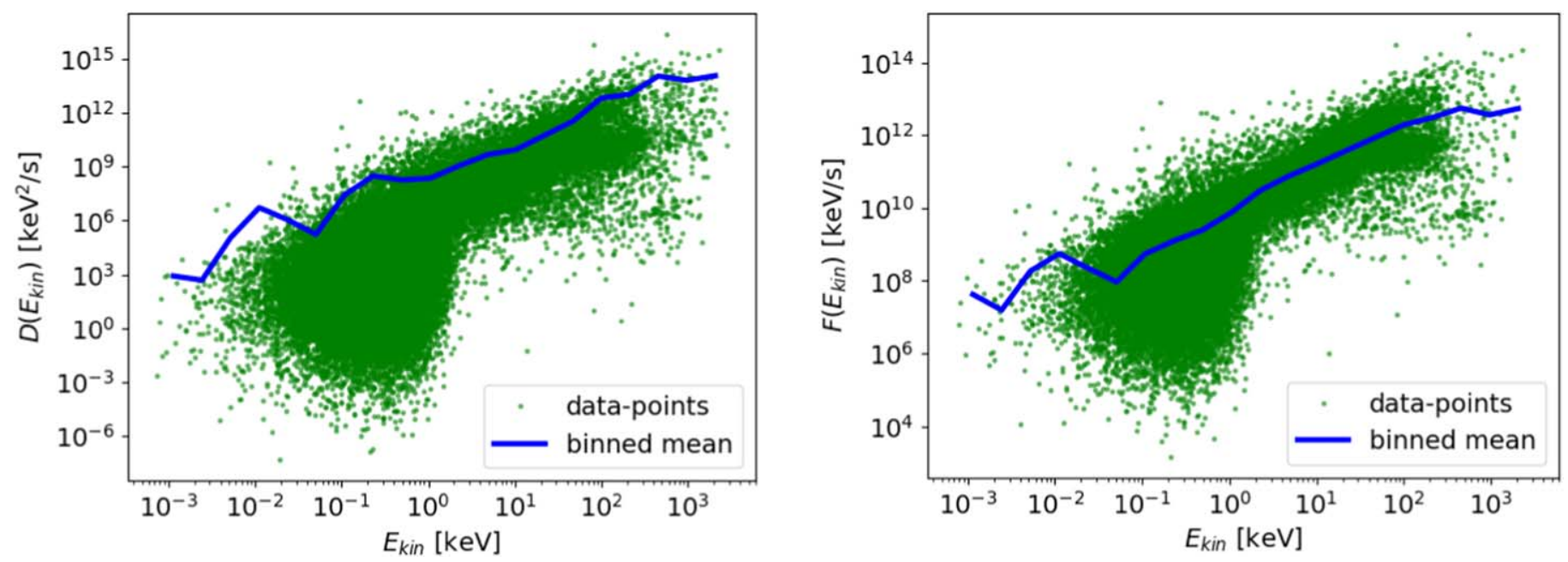

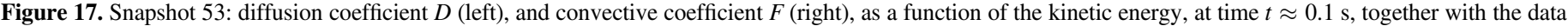
points on which the binned statistics are based.

(see Ragwitz \& Kantz 2001), can then be determined by applying binned statistics, i.e., by calculating the mean values for each energy bin separately at a given time instance $t_{k}$.

Figure 17 shows the diffusion and drift coefficient for snapshot 53 at $t=t_{100} \approx 0.1 \mathrm{~s}$, together with the data points that form the sample of the binned statistics $\left(\epsilon_{j}\left(t_{100}\right)^{2} /\right.$ $2\left(t_{k+h}-t_{k}\right)$ and $\epsilon_{j}\left(t_{100}\right) /\left(t_{k+h}-t_{k}\right)$, respectively, as a function of $\left.E_{k i n, j}\left(t_{100}\right)\right)$. The binned mean for both coefficients exhibits a quite clear power-law functional form, yet the spread of the data points around the mean values is large, namely several orders of magnitude, which is a first hint that the estimate of the transport coefficients is problematic.

To clarify the situation further, we show in Figure 18 the histogram $p(\epsilon)$ of the energy increments $\epsilon_{j}$, which follows a double power-law distribution, with index -1.97 at the highest energies. It then follows that the drift coefficient $F$, as a mean value of the increments, is not representative of the scale-free data, and the diffusion coefficient $D$, as a variance of the increments, is ill-defined. This result, namely that the Fokker-Planck formalism breaks down, has been found also for cases of strong turbulence (Isliker et al. 2017b) and of turbulent reconnection (Isliker et al. 2017a).

\subsection{Fractional Transport Equation (FTE)}

The power-law tail of the distribution of energy increments implies that the particle dynamics is anomalous. With occasionally large energy steps being made, the particles perform Levy-flights in energy space when their dynamic is interpreted as a random walk. In Isliker et al. (2017b), we introduced a formalism for a FTE that is able to cope with this kind of non-classical dynamics. In this approach the distribution of energy increments is interpreted as a symmetric stable Levy distribution $p_{\epsilon}(\epsilon ; \alpha, a)$, which is defined in Fourier space (characteristic function, $\epsilon \rightarrow k)$ as $\hat{p}_{\epsilon}(k ; \alpha, a)=\exp \left(-a|k|^{\alpha}\right)$, and which has a power-law tail in energy space, $p_{\epsilon}(\epsilon ; \alpha$, a) $\sim \epsilon^{-(1+\alpha)}$ (see e.g., Hughes 1995). The energy increments are sampled over constant time intervals $\Delta:=t_{k+h}-t_{k}$, which 

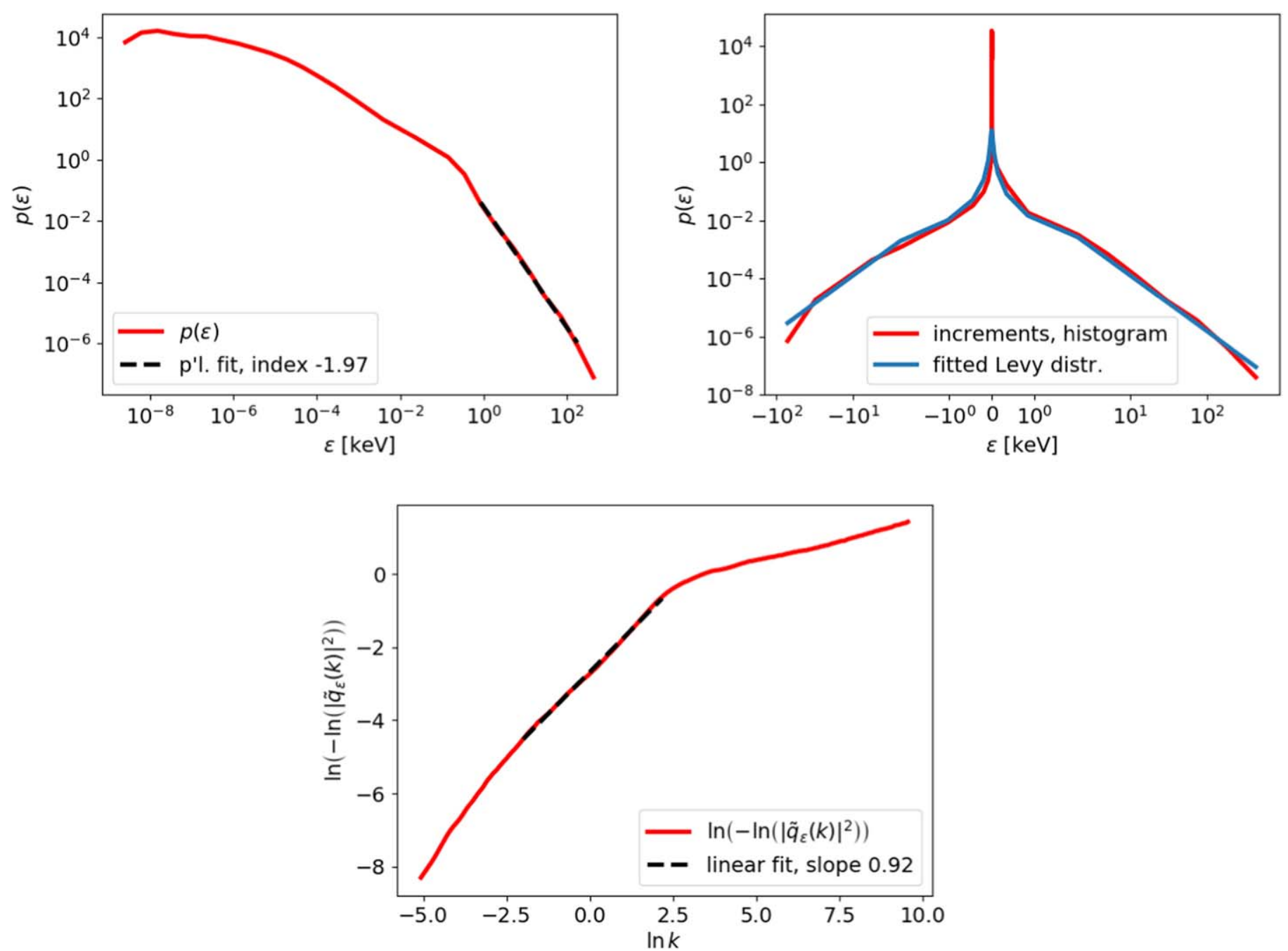

Figure 18. Snapshot $53, t \approx 0.1 \mathrm{~s}$. (a) Distribution of energy increments. (b) Two-sided distribution of energy increments, together with the fitted stable Levy distribution. (c) Characteristic function estimate.

has as the consequence that the temporal part of the FTE is non-fractional.

The FTE has the form (for details and its derivation see Isliker et al. 2017b)

$$
\frac{\partial f}{\partial t}=\frac{a}{\Delta} D_{|E|}^{\alpha} f-\frac{f}{\tau_{\mathrm{esc}}},
$$

with $D_{|E|}^{\alpha}$ being the symmetric Riesz fractional derivative of order $\alpha$ (defined in Fourier space as $\left.\mathcal{F}\left(D_{|E|}^{\alpha} f\right)=-|k|^{\alpha} \hat{f}\right)$, $a$ being the constant of the Levy stable distribution that is related to the width of the distribution of increments, and $\Delta$ being the applied time step in monitoring the particles' energy increments, defined just above.

With $\Delta$ already given, we still need to determine two parameters, $\alpha$ and $a$. A first way to infer $\alpha$ is through the index of the power law in the tail of the distribution of energy increments, which yields $\alpha=0.97$; see Figure 18. A second way to determine $\alpha$ and also $a$ is through the characteristic function method (e.g., Borak et al. 2005; Koutrouvelis 1980), as described in Isliker et al. (2017b), which gives $\alpha=0.92$ and $a=0.03$; see Figure 18. A third way to infer the parameters is by applying a maximum-likelihood estimate (e.g., Borak et al. 2005), which for financial data is known to be the most precise

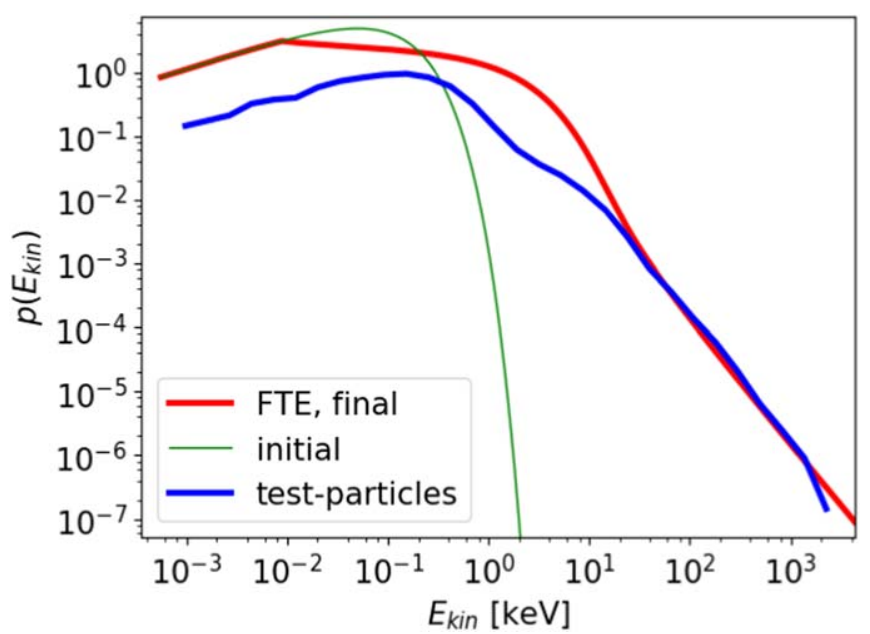

Figure 19. Snapshot 53: kinetic energy distribution at initial and final times $(t \approx 0.1 \mathrm{~s})$ from the test-particle simulations, together with the solution of the FTE at the final time.

method. In our application, however, we find large deviations when comparing to the index of the power-law tail in the increments, as the method yields $\alpha=1.24, a=3.2$. The fit of 

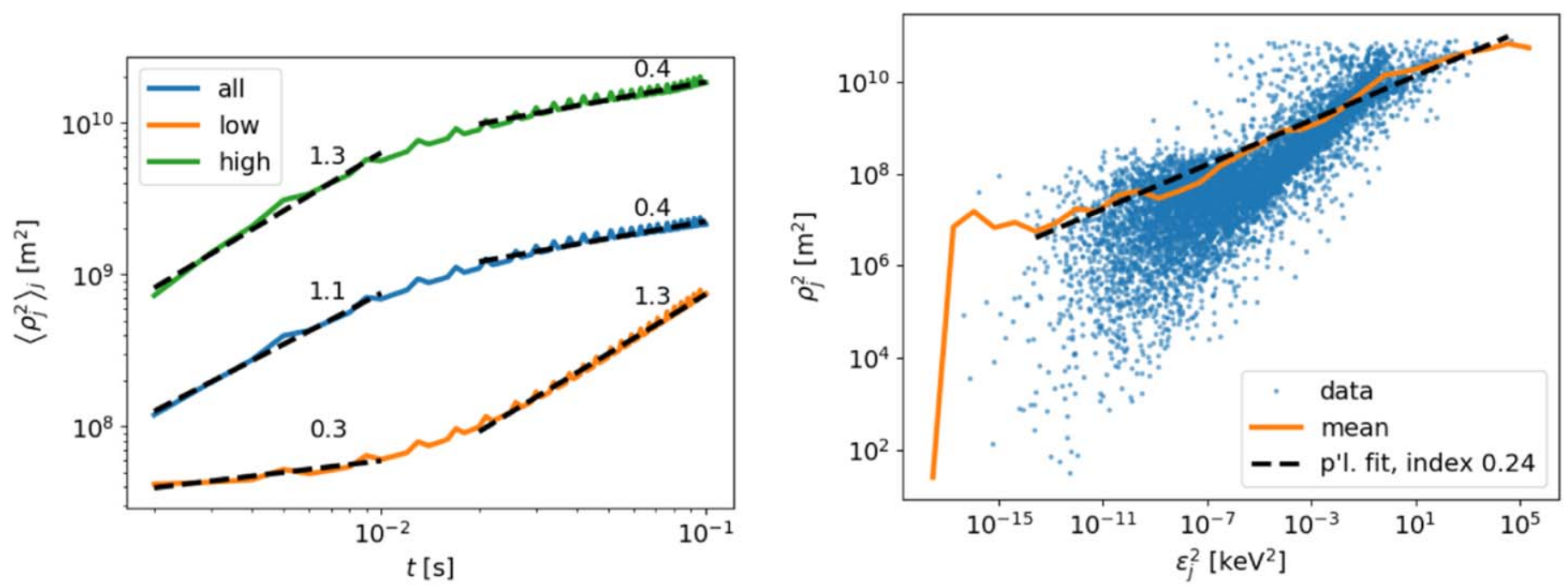

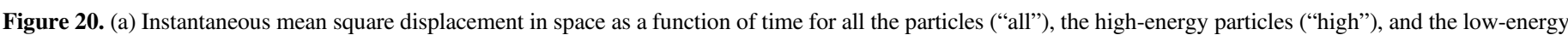

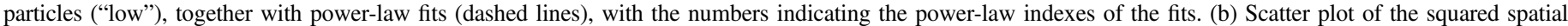
increments $\rho_{j}^{2}$ as a function of the squared energy increments $\epsilon_{j}^{2}$, together with the binned mean and a power-law fit to the latter.

the Levy stable distribution is shown in Figure 18, with the parameters estimated through the characteristic function method, and it indeed is in good agreement with the distribution in the power-law tail.

For the numerical solution of the FTE, we use the Grünwald Letnikov definition of fractional derivatives (see, e.g., Kilbas et al. 2006), in the matrix formulation of Podlubny et al. (2009), and in order to allow for a logarithmically equally spaced grid on the energy axis, we make use of the formulation for non-equidistant grid points in Podlubny et al. (2013). Timestepping is done by the backward Euler method. The solution of the FTE at the same final time as for the test particles is shown in Figure 19, there is very good coincidence with the distribution function from the test-particle simulation for the entire power-law tail. The FTE is thus an adequate transport model for the acceleration process of the high-energy, powerlaw-distributed particles. We note that modeling transport in energy space isolated from the simultaneous spatial transport, despite being of valuable interest, is a simplification. For a full understanding of the dynamics, a model for the combined transport in energy and position space is needed. However, such a model first needs to be developed, which seems to be a nontrivial problem for our case here of doubly anomalous transport (see also the next section).

\section{Spatial Diffusion}

We define the spatial mean square displacement (MSD) as

$$
\left\langle\rho_{j}^{2}\right\rangle:=\left\langle\left(\boldsymbol{x}_{j}\left(t_{k+h}\right)-\boldsymbol{x}_{j}\left(t_{k}\right)\right)^{2}\right\rangle_{j},
$$

i.e., we formally follow the definition of the displacements in energy, Equation (14), which explicitly allows for a timedependent MSD.

The MSD is shown in Figure 20. The particles are moderately super-diffusive in the initial phase up to $0.01 \mathrm{~s}$, and then they turn over and become clearly sub-diffusive. Also, we have separated the particles into two populations, one that reaches high energies and forms the tail of the distribution, $E_{k i n}>20 \mathrm{keV}$, and one that corresponds to the low-energy, bulk population, $E_{k i n}<20 \mathrm{keV}$. The high-energy population shows a very similar behavior as the entire sample of particles; it just is more clearly super-diffusive at small times. The lowenergy particles, on the other hand, are sub-diffusive at small times, and then turn to super-diffusive at large times. Thus, their behavior is the inverse of that of the high-energy particles.

Figure 20 also shows the increments $\rho_{j}^{2}$ as a function of the corresponding energy increments $\epsilon_{j}^{2}$. There is a correlation between the two kind of increments, with a power-law shaped functional dependence; the spread though of the data points around this power-law is rather large. There thus is a trend for large energy increments to be associated with large spatial increments.

\section{Discussion and Conclusions}

Using a 3D resistive MHD code, Archontis \& Hood (2013) simulated the emergence and eruption of solar magnetic fields interacting with a unipolar magnetic field. Their analysis was concentrated on the macroscopic parameters during the formation and emission of a standard and a more explosive blowout jet. A key element in their analysis was the formation and the evolution of the reconnecting current sheet at the base of the jet, where the emerging magnetic flux meets the ambient magnetic field. We use their numerical results for the electromagnetic fields to analyze the heating and acceleration of particles in the vicinity of the reconnecting current sheets and the characteristics of the particles escaping from the energy release volume. The interaction of the emerging flux with the ambient magnetic field is evolving on scales of tenths of minutes and ignites the standard jet 30 minutes after the initiation of the numerical experiment. The blowout jet reaches its peak of activity about 20 minutes later. The acceleration time of the electrons, in the presence of the electromagnetic fields of the MHD simulations, is much faster; their energy distribution reaches saturation in less than one second. In the analysis presented here, we study the statistical properties of the electric field at two particular times, $t=30 \mathrm{~m}$ and $t=53 \mathrm{~m}$, i.e., during the formation of the standard and the blowout jet, and we investigate the kinetic evolution of electrons at these two times. 
The electric fields spontaneously develop a fragmented and fractal structure in the spatial vicinity of the reconnecting current sheet, and the clusters constituting the fractal obey power-law size distributions. The probability distribution of the electric field and its parallel component, the parallel electric field's energy density, and the MHD energies, all exhibit power-law tails.

The electrons interacting with the fragmented electric fields are heated and accelerated near the base of the jet at the times where the standard and blowout jets are formed. The highest energies reached are larger by roughly a factor of two at the blowout jet than at the standard jet, and in both cases the power-law index of the tail of the energy distribution is evolving in time. The heating of the particles at low energies is rather gradual, reaching saturation at a temperature of $4.5 \mathrm{MK}$ (see the observations reported by Bain \& Fletcher 2009). The escaping high-energy particles form a super-hot population with temperature $150 \mathrm{MK}$, and with a power-law tail (Glesener et al. 2012; Glesener \& Fleishman 2018; Chen et al. 2013).

The acceleration is a fast, one-step process that typically lasts about $10 \mathrm{~ms}$. There is no random-walk-like behavior, so there is no Fermi process operating, contrary to the widely assumed scenarios reported in the current literature (Drake et al. 2006, 2013; Kowal et al. 2011; Guo et al. 2015; Lazarian et al. 2015) for the acceleration of particles in fragmented current sheets. The heating mechanism also does not follow a random-walk process but is rather of a systematic drift nature.

The acceleration of the electrons is exclusively in the parallel direction and is clearly and solely caused by the parallel electric field, whose statistical properties in the vicinity of the unstable current sheet thus play the key role in the characteristics of the heated and accelerated particles. The power-law distributions of both, the strengths of the parallel electric field, and the sizes of the clusters that it forms, act together in the acceleration of the particles, whereby none of their two power-law indices can be expected to be directly mapped onto the indices of the tail of the electrons' kinetic energy distributions, as the system is not linear and exhibits nontrivial complex dynamics, and there is also a dynamic effect, namely the particles' Lagrangian viewpoint, i.e., the electric fields a particle witnesses along its trajectory depend on the initial conditions.

Analyzing the transport properties of the accelerated particles in energy space, we have shown that the FokkerPlanck transport equation is not consistent with the test-particle dynamics inside the simulation box for two reasons: (1) the transport coefficients cannot be determined from the particle dynamics in a meaningful way, and (2) the random-walk picture that is inherent in the FP approach does not apply. Of course, mathematically there may exist functional forms of transport coefficients that would reproduce the observed energy distribution when inserted into the FP equation. However, they would just be formal and completely lack a consistent physical interpretation as diffusion and convection coefficients. We have also shown that an FTE (Isliker et al. 2017a, 2017b) is able to reproduce the acceleration process. Its coefficients can consistently be determined from the particle dynamics, but despite its success in reproducing the particles' acceleration, there still is an inconsistency in its theoretical justification, since in its derivation it is also assumed that a random-walk process (of the Levy type) takes place; see Isliker et al. (2017b). A completely adequate transport model for the case of single, power-law-dominated and fractal acceleration, does not seem to exist, to our knowledge.

The spatial transport of the heated plasma and the accelerated particles is anomalous, as one would expect for the transport of particles in a fractal environment of electric fields. The high-energy particles execute super diffusion in the initial phase of their acceleration and later become subdiffusive. The opposite is true for the low-energy, just heated particles, which start as sub-diffusive and end up in the final stage as super-diffusive. The squared spatial increments of the electrons increase with increasing squared energy increments, i.e., large spatial displacements are correlated with large energy gains. We must emphasize that the combined and simultaneous particle transport (in position- and energy space) inside a fractally distributed electric field environment, with the strength of the electric field moreover being power-law-distributed, is a complex problem that requires further analysis for an understanding of its nature.

The scenario emerging from the analysis presented here is related very nicely with current observations of energetic particles during the formation and eruption of jets. Initially, there is a slow evolution of the emerging flux until the point where the reconnecting large-scale current sheet is formed (Archontis \& Hood 2013; Jiang et al. 2016). The spontaneous fragmentation of the current sheet creates a very efficient environment for the heating and acceleration of particles in the vicinity of the base of the standard and blowout jets. The highenergy emission recorded by the RHESSI satellite in the vicinity of jets confirms the presence of super-hot plasmas, as we find it in our model. Also, the simultaneous detection of type III and HXR bursts during impulsive explosions, and the correlations with solar energetic particles, are related with jets (Bain \& Fletcher 2009; Glesener et al. 2012; Chen et al. 2013; Raouafi et al. 2016; Glesener \& Fleishman 2018; Archontis \& Vlahos 2019), and can directly be understood in the frame of our model as resulting from the acceleration on sub-second timescales in the fragmented environments near jets, when embedded in the global topology of emerging flux, as in Figures 2 or 3.

The results reported here are based on the coupling of a resistive MHD code with a test-particle code. This analysis has several advantages, under the prerequisite that the energy transfer from the electromagnetic fields to the heated and accelerated particles is limited to less than $15 \%-20 \%$ of the MHD energies. Also, no temporal interpolation is needed if the evolution of the MHD fields is very slow compared to the particle acceleration time. The analysis is indeed very useful because the statistical characteristics and the transport properties of particles can be analyzed on the kinetic level, and one can trace the evolution of the particle energy distribution in realistic, large-scale, open systems. The main disadvantage is that test-particle simulations cannot estimate the feedback of the accelerated particles on the evolution of the electromagnetic fields; therefore the details of some of the quantitative results (power-law index of the high-energy tail, maximum energy reached, acceleration time) of the saturated particle distributions must be expected to be revised to some degree when feedback is included. On the other hand, the basic statistical and transport properties studied here will persist and become a useful guide for the development of second-generation coupled MHD and PIC codes for large-scale, open systems. These codes will be able to treat even more realistically particle 
heating and acceleration in the case of large-scale explosions in the solar atmosphere. Several groups are working toward this new generation of codes that couple MHD and PIC simulations, but it is too early to evaluate their success (Chen et al. 2017; Drake et al. 2018; Makwana et al. 2018; van Marle et al. 2018).

L.V. was partly supported by the European Union (European Social Fund) and the Greek national funds through the Operational Program "Education and Lifelong Learning" of the National Strategic Reference Frame Work Research Funding Program: Thales. Investing in Knowledge Society through the European Social Fund. V.A. acknowledges support by the Royal Society.

\section{ORCID iDs}

H. Isliker (i) https://orcid.org/0000-0001-9782-2294

V. Archontis (i) https://orcid.org/0000-0002-6926-8676

L. Vlahos 1 h https://orcid.org/0000-0002-8700-4172

\section{References}

Arber, T. D., Longbottom, A. W., Gerrard, C. L., \& Milne, A. M. 2001, JCoPh, 171,151

Archontis, V. 2012, RSPTA, 370, 3088

Archontis, V., \& Hood, A. W. 2012, A\&A, 537, A62

Archontis, V., \& Hood, A. W. 2013, ApJL, 769, L21

Archontis, V., Hood, A. W., \& Tsinganos, K. 2013, ApJ, 778, 42

Archontis, V., Moreno-Insertis, F., Galsgaard, K., Hood, A., \& O’Shea, E. 2004, A\&A, 426, 1047

Archontis, V., Moreno-Insertis, F., Galsgaard, K., \& Hood, A. W. 2005, ApJ, 635,1299

Archontis, V., \& Vlahos, L. 2019, RSPTA, 377, 20190152

Bain, H. M., \& Fletcher, L. 2009, A\&A, 508, 1443

Baumann, G., Haugbølle, T., \& Nordlund, A. 2013, ApJ, 771, 93

Borak, S., Härdle, W., \& Weron, R. 2005, in Statistical Tools for Finance and Insurance, ed. P. Cizek, W. Härdle, \& R. Weron (Berlin: Springer), 21

Cargill, P., Vlahos, L., Baumann, G., Drake, J., \& Nordlund, Å. 2012, SSRv, 173,223

Chen, N., Ip, W.-H., \& Innes, D. 2013, ApJ, 769, 96

Chen, Y., Tóth, G., Cassak, P., et al. 2017, JGRA, 122, 10318

Dahlin, J. T., Drake, J. F., \& Swisdak, M. 2016, PhPl, 23, 120704

Drake, J. F., Arnold, H., Swisdak, M., \& Dahlin, J. T. 2018, PhPl, 26, 012901

Drake, J. F., Swisdak, M., Che, H., \& Shay, M. A. 2006, Natur, 443, 553
Drake, J. F., Swisdak, M., \& Fermo, R. 2013, ApJL, 763, L5

Galsgaard, K., Moreno-Insertis, F., Archontis, V., \& Hood, A. 2005, ApJL, 618, L153

Glesener, L., \& Fleishman, G. D. 2018, ApJ, 867, 84

Glesener, L., Krucker, S., \& Lin, R. P. 2012, ApJ, 754, 9

Grebogi, C., \& Littlejohn, R. G. 1984, PhFl, 27, 1996

Guo, F., Liu, Y.-H., Daughton, W., \& Li, H. 2015, ApJ, 806, 167

Hamamatsu, K., Takizuka, T., Hayashi, N., \& Ozeki, T. 2007, PPCF, 49, 1955

Heyvaerts, J., Priest, E. R., \& Rust, D. M. 1977, ApJ, 216, 123

Holman, G. D. 1985, ApJ, 293, 584

Hoshino, M. 2012, PhRvL, 108, 135003

Hughes, B. D. 1995, Random Walks and Random Environments, Vol. 1: Random Walks (Oxford: Clarendon)

Isliker, H., Pisokas, T., Vlahos, L., \& Anastasiadis, A. 2017a, ApJ, 849, 35

Isliker, H., Vlahos, L., \& Constantinescu, D. 2017b, PhRvL, 119, 045101

Jiang, C., Wu, S. T., Feng, X., \& Hu, Q. 2016, NatCo, 7, 11522

Karimabadi, H., \& Lazarian, A. 2013, PhPl, 20, 112102

Karney, C. F. 1986, CoPhR, 4, 183

Kilbas, A., Srivastava, H., \& Trujillo, J. 2006, Theory and Applications of Fractional Differential Equations (Amsterdam: Elsevier)

Koutrouvelis, I. A. 1980, Journal of the American Statistical Association, 75,918

Kowal, G., de Gouveia Dal Pino, E. M., \& Lazarian, A. 2011, ApJ, 735, 102

Lazarian, A., Eyink, G., Vishniac, E., \& Kowal, G. 2015, RSPTA, 373, 20140144

Lazarian, A., Vlahos, L., Kowal, G., et al. 2012, SSRv, 173, 557

le Roux, J., Zank, G. P., \& Khabarova, O. V. 2018, ApJ, 864, 158

le Roux, J. A., Zank, G. P., Webb, G. M., \& Khabarova, O. 2015, ApJ, 801,112

Li, X., Guo, F., Li, H., \& Birn, J. 2018, ApJ, 855, 80

Makwana, K. D., Keppens, R., \& Lapenta, G. 2018, PhP1, 25, 082904

Moore, R. L., Cirtain, J. W., Sterling, A. C., \& Falconer, D. A. 2010, ApJ, 720,757

Moreno-Insertis, F., \& Galsgaard, K. 2013, ApJ, 771, 20

Onofri, M., Isliker, H., \& Vlahos, L. 2006, PhRvL, 96, 151102

Podlubny, I., Chechkin, A., Skovranek, T., Chen, Y., \& Vinagre Jara, B. M. 2009, JCoPh, 228, 3137

Podlubny, I., Skovranek, T., Vinagre Jara, B. M., et al. 2013, RSPTA, 371, 20120153

Ragwitz, M., \& Kantz, H. 2001, PhRvL, 87, 254501

Raouafi, N. E., Patsourakos, S., Pariat, E., et al. 2016, SSRv, 201, 1

Tao, X., Chan, A. A., \& Brizard, A. J. 2007, PhPl, 14, 092107

Turkmani, R., Cargill, P. J., Galsgaard, K., Vlahos, L., \& Isliker, H. 2006, A\&A, 449, 749

van Marle, A. J., Casse, F., \& Marcowith, A. 2018, MNRAS, 473, 3394

Wyper, P. F., Antiochos, S. K., \& DeVore, C. R. 2017, Natur, 544, 452

Wyper, P. F., DeVore, C. R., Karpen, J. T., \& Lynch, B. J. 2016, ApJ, 827, 4

Zank, G. P., le Roux, J. A., Webb, G. M., Dosch, A., \& Khabarova, O. 2014, ApJ, 797, 28 